\title{
CHD8 Suppression Impacts on Histone H3 Lysine 36 Trimethylation and
}

\section{Alters RNA Alternative Splicing.}

\author{
Emanuela Kerschbamer, ${ }^{*}$ Takshashila Tripathi, $^{1 *}$ Serkan Erdin, ${ }^{2,3}$ Elisa Salviato, ${ }^{4}$ \\ Francesca Di Leva, ${ }^{1}$ Endre Sebestyén, ${ }^{4}$ Michele Arnoldi, ${ }^{1}$ Matteo Benelli, ${ }^{5}$ James F. \\ Gusella, ${ }^{2,3,6}$ Silvano Piazza, ${ }^{7}$ Francesca Demichelis, ${ }^{8}$ Michael E. Talkowski, ${ }^{2,3,6}$ Francesco \\ Ferrari, ${ }^{4,9}$ and Marta Biagioli ${ }^{1, \#}$
}

${ }^{1}$ Neuroepigenetics laboratory, Department of Cellular, Computational and Integrative Biology, (CIBIO) University of Trento, Trento, Italy

${ }^{2}$ Center for Genomic Medicine, Massachusetts General Hospital, Boston, MA, United States

${ }^{3}$ Program in Medical and Population Genetics, Broad Institute, Cambridge, MA, United States

${ }^{4}$ IFOM, the FIRC Institute of Molecular Oncology, Milan, Italy

${ }^{5}$ Bioinformatics Unit, Hospital of Prato, Istituto Toscano Tumori, Prato, Italy

${ }^{6}$ Department of Neurology, Harvard Medical School, Boston, MA, United States

${ }^{7}$ Bioinformatic facility, Department of Cellular, Computational and Integrative Biology (CIBIO) University of Trento, Italy

${ }^{8}$ Laboratory of Computational and Functional Oncology, Department of Cellular, Computational and Integrative Biology (CIBIO), University of Trento, Trento, Italy

${ }^{9}$ CNR Institute of Molecular Genetics "Luigi Luca Cavalli-Sforza", Pavia, Italy

* Joint co-first authors

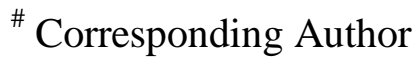

Running Title: CHD8 Suppression alters RNA Alternative Splicing

Key words: Autism Spectrum Disorder, CHD8, Chromatin, H3K36me3, Alternative Splicing 


\begin{abstract}
Disruptive mutations in the chromodomain helicase DNA binding protein 8 (CHD8) have been recurrently associated with Autism Spectrum Disorders (ASD). In normal cellular physiology, CHD8 co-purifies with MLL1 and MOF transcriptional activation complex, with elongating RNAPII and directly binds to DNA promoters and enhancers regions, thus a regulatory role in transcriptional initiation and elongation could be postulated.
\end{abstract}

Here we investigated how chromatin landscape reacts to $C H D 8$ suppression by analyzing a panel of histone modifications in induced pluripotent stem cell-derived neural progenitors. We interrogated transcriptionally active and repressed regions, as well as active and poised enhancers.

CHD8 suppression led to significant reduction (47.82\%) in histone H3K36me3 peaks at gene bodies, particularly impacting on transcriptional elongation chromatin states. H3K36me3 reduction specifically affects highly expressed, CHD8-bound genes. Histone H3K36me3 reduction associated to $C H D 8$-suppression does not functionally impact on global transcriptional levels, but correlated with altered alternative splicing patterns of $\sim 2000$ protein coding genes implicated in "RNA splicing", "mitotic cell cycle phase transition" and "mRNA processing", especially affecting alternative first exon and exon skipping events.

In summary, our results point toward broad molecular consequences of CHD 8 suppression, implicating altered histone deposition/maintenance and RNA processing regulation as important regulatory processes in ASD. 


\section{Introduction}

De novo truncating mutations in CHD 8 have been reported and independently validated to be a strong risk factor for autism spectrum disorders (ASD) (Neale et al. 2012; O'Roak et al. 2012a; O'Roak et al. 2012b; Talkowski et al. 2012; Iossifov et al. 2014; Parikshak et al. 2013; De Rubeis et al. 2014). CHD8 has been classified as a high confidence ASD candidate risk factor (score 1) in the Simons Foundation Autism Research Initiative (SFARI) [https://gene.sfari.org (Abrahams et al. 2013)] with most CHD 8 mutations, identified from $\sim 70$ ASD probands (www.ncbi.nlm.nih.gov/clinvar/ interrogated on January $\left.8^{\text {th }}, 2020\right)$, considered disruptive and resulting in protein haploinsufficiency. $C H D 8$ defines a subclass of ASD patients, displaying evident macrocephaly, distinct faces, sleep problems and gastrointestinal complaints (Bernier et al. 2014; Yasin et al. 2019). Most of these phenotypic characteristics were recapitulated in $c h d 8$ knock-down zebrafish (Bernier et al. 2014; Sugathan et al. 2014) and, more recently, in Chd8 suppression mouse models (Durak et al. 2016; Katayama et al. 2016; Suetterlin et al. 2018). Indeed, chd8-morpholino zebrafish and $C h d 8$ heterozygous mice display increased brain size, due to increased numbers of proliferating cells and newborn neurons, possibly initiated by altered gene expression in the developing neocortex (Sugathan et al. 2014; Suetterlin et al. 2018). Remarkably, genome-wide transcriptomic changes that impact on ASD related genes were also detected in vitro, in human neural progenitor cells (NPC) with reduced CHD8 expression (Sugathan et al. 2014). Taken together, these observations suggest that aberrant genome-wide transcription leading to altered brain development is strictly correlated to reduced levels of CHD8 function. However, the detailed molecular mechanism through which CHD8 regulates this process still remains obscure. 
A direct effect can be proposed since CHD8 is able to bind DNA at promoters and enhancer regions in hNPCs, mouse midfetal brain and embryonic cortex (Sugathan et al. 2014; Cotney et al. 2015). However, an indirect mechanism can also be postulated since other genes, not bound by CHD8, appear also to be transcriptionally dysregulated following CHD8 suppression (Sugathan et al. 2014). Chromatin structure is intimately related to transcription, as DNA tightly packed around nucleosomes prevents transcription, while, conversely, the exchange or removal of nucleosomes allows free access to DNA, thus correlating with active gene expression (Venkatesh and Workman 2015). Using mass spectrometry, CHD8 was shown to copurify with components of the MLL and CoREST, SWI/SNF and NuRD ATP-dependent remodeling complexes, supporting its possible role in transcriptional initiation (Thompson et al. 2008). On the other hand, CHD1, another member of the CHD protein family, has been shown to interact with the PAF1 transcription elongation complex maintaining H3K4me3/H3K36me3 domains at actively transcribed genes (Lee et al. 2017). Reduction of CHD1 alters H3K4me3 and H3K36me3 patterns, suggesting a role for CHD1 in establishing/maintaining the boundaries of these mutually exclusive histone marks (Lee et al. 2017). The SETD2 and SETD5 histone H3, Lysine 36 methyltransferases normally associate with RNAPII, and their activity results in increased H3K36me3 toward the 3' end of active genes (Krogan et al. 2003; Kizer et al. 2005). Loss of SETD2/5-dependent H3K36me in mammals (Sessa et al. 2019) as well as loss of yeast Chd1 causes reduced Rpd3S activity (histone deacetylase complex), increased acetylation, and increased cryptic transcription within gene bodies (Selth et al. 2010). Based on its placement on the phylogenetic tree and the presence of an ATPase domain (Thompson et al. 2008), CHD8 is most likely acting as an ATP-dependent chromatin remodeling factor; thus, similar to CHD1, CHD8 loss might cause increased nucleosome turnover and alterations in co-transcriptional 
processes, such as cryptic transcription within gene bodies and alternative splicing (RadmanLivaja et al. 2012; Smolle et al. 2012).

Alternative splicing (AS), one of the major contributor to protein diversity in eukaryotes, is important for highly specialized cells, such as neurons. Aberrant splicing might contribute to neuronal dysfunction and has been associated with several neurological diseases (Cuajungco et al. 2003; Mordes et al. 2006) (Wang et al. 2012; Gompers et al. 2017). Broad chromatin conformation and transcriptional kinetics are major factors in the regulation of AS. Chromatin relaxation accelerates RNAPII processing and correlates with alternative exons skipping; conversely, packed nucleosomes slow down RNAPII progression causing pausing of transcription and the inclusion of non-constitutive weak exons (Luco et al. 2010; Nilsen and Graveley 2010; Luco and Misteli 2011). As experimental evidence is pointing to dysregulated chromatin regulation as a key feature in the pathogenesis of ASD, it is tempting to hypothesize that chromatin function of CHD proteins, and CHD8 in particular, might act to regulate RNA transcription, elongation and processing thereby being responsible for the characteristic neurodevelopmental effects observed in ASD.

In order to possibly dissect this mechanism, we characterized the consequences of $C H D 8$ suppression on the chromatin landscape, analyzing different histone modifications using chromatin immunoprecipitation and sequencing (ChIP-seq). Specifically, we interrogated histone marks characteristic of transcriptionally active (H3K4me2, H3K4me3, H3K27ac, H3K36me3) and repressed regions (H3K27me3) as well as active enhancers (H3K4me1, H3K27ac) in control induced pluripotent stem cell (iPSC)-derived neuronal progenitors cell line and in previously characterized lines where a $\sim 50 \%$ reduction in $C H D 8$ was obtained by lentiviral delivery of shRNAs (Sugathan et al. 2014). We uncovered alterations affecting the H3K36me3 histone mark 
in the body of highly transcribed genes, which do not primarily affect RNA transcription, but rather alter alternative splicing of genes implicated in "histone modification", "covalent chromatin modification" and "mitotic cell cycle phase transition", thus unveiling altered histone deposition/maintenance and RNA processing regulation as important regulatory processes in ASD. 


\section{Results}

Epigenetic consequences of CHD8 suppression in human neuronal progenitor cells

To assess the functional consequences of $C H D 8$ suppression in chromatin organization, we resourced to previously characterized control iPSC-derived neuronal progenitors cell line, GM8330-8 and its derivatives where $\sim 50 \%$ reduction in CHD8 was obtained by lentiviralmediated delivery of short-hairpin RNAs (shRNAs) (Sugathan et al. 2014). In these model systems, we analyzed six different histone modifications using chromatin immunoprecipitation and sequencing (ChIP-seq), specifically interrogating transcriptionally active (H3K4me2, H3K4me3, H3K27ac, H3K36me3) and repressed regions (H3K27me3) as well as active enhancers (H3K4me1, H3K27ac). For each of the six histone marks, three independent shRNAs targeting the coding sequence of $C H D 8(\mathrm{Sh} 1, \mathrm{Sh} 2, \mathrm{Sh} 4)$ and two technical replicate controls against GFP sequence were used (Fig. 1A). Sh1-CHD8, Sh2-CHD8, Sh4-CHD8 presented nearly comparable levels of CHD8 at $~ 50 \%$ of the physiological levels, thus precisely mimicking the human haploinsufficiency condition (Sugathan et al. 2014). Importantly, for CHD8-knock-down models as well as for GFP controls, genome-wide transcriptomic data and CHD8 binding sites were available (Fig. 1A) (Sugathan et al. 2014).

ChIP-seq experiments were conducted to obtain on average 40M reads for narrow marks (H3K4me3/me2/me1, H3K27ac) and 60M for broad histone marks (H3K36me3/K27me3) and for INPUT samples. After mapping and filtering, an average of 42308 peaks per sample was identified (Suppl. Fig. 1A-B), and showed the expected enrichment pattern and metagene profiles at the transcriptional start site (H3K4me3/me2/me1, H3K27ac), gene body (H3K36me3) of actively transcribed genes or on larger genomic regions spanning transcriptionally silent gene 
units (H3K27me3) and the expected enrichment correlation with ENCODE public datasets (Suppl. Fig. 1C-D) (Consortium 2012; Davis et al. 2018).

Upon CHD8 suppression, H3K4me1, H3K27ac and H3K36me3 presented a substantial decrease in the number of peaks $(37.44 \%, 38.45 \%$ and $47.82 \%$, respectively) compared to control (intersection of Sh-GFP and Sh-GFP2) (Fig. 1B-C). Peaks detected exclusively in knock-down replicates (intersection of Sh2-CHD8 and Sh4-CHD8, the two Sh-CHD8 presenting nearly identical (46.63-48.61\%) levels of CHD8 suppression (Fig. 2C-D)) were limited (Fig. 1C). Notably, the third biological replicate Sh1-CHD8, presenting lower levels of CHD8 suppression (Fig. 2C-D) (Sugathan et al. 2014), generally confirmed previous findings and the intersection of two biological replicates - in any of the possible combinations (Sh1-Sh2 CHD8, Sh2-Sh4 CHD8, Sh1-Sh4 CHD8), supported the reduction in H3K4me1, H3K27ac and H3K36me3 enrichment peaks (Suppl. Fig. 2A). In addition, Sh2-CHD8 and Sh-GFP independent ChIP-seq datasets (generated in different laboratory than previous set) again sustained the conclusion that CHD 8 suppression was associated with a decreased $\mathrm{H} 3 \mathrm{~K} 4 \mathrm{me} 1$ and H3K36me3 enrichment (Suppl. Fig. 2B).

Genes presenting reduced peaks for H3K4me1, H3K27ac and H3K36me3 histone marks were involved in "protein targeting", "ribosome biogenesis" and "mitotic nuclear division" pathways (Fig. 1D) and enriched for 'essential genes' (required for a cell's survival, Wang et al. 2015) and 'constrained' genes (intolerant to loss of function mutations, gnomAD), 'FMRP targets in brain' (Darnell et al. 2011), 'post synaptic genes' (Krishnan et al. 2016), and the 'M3 co-expression module' (Parikshak et al. 2013), whose expression peaks early during nervous system development (Fig. 1E). 


\section{CHD8 suppression significantly affects transcriptional elongation chromatin states}

By combined analysis of the histone marks enrichment through ChromHMM (Ernst and Kellis 2012), we defined 10 types of genomic regions with a specific chromatin state in control hiNPC (1. transcriptional initiation (H3K4me3, H3K4me2, H3K4me1, H3K36me3), 2. transcriptional elongation (high H3K36me3), 3. weak transcribed (low H3K36me3), 4. strong enhancer (H3K4me2, H3K4me1, H3K27ac), 5. weak/poised enhancer a (H3K4me2, H3K4me1), 6. weak/poised enhancer b (H3K4me1, H3K27ac), 7. active promoter (H3K4me3, H3K4me2, H3K4me1, H3K27ac), 8. inactive/poised promoter (H3K27me3, H3K4me3, H3K4me2, H3K4me1), 9. Polycomb repressed (H3K27me3), 10. heterochromatin/low signal (no enrichment)) (Fig. 2 A-left). To identify states affected by CHD 8 knock-down, we then compared the histone marks (peak counts for each histone mark within each chromatin states) across two conditions, i.e. controls versus CHD8 knock-down (Sh2-CHD8 and Sh4-CHD8) (Fig. 2 A-right) and detected transcriptional elongation, strong-weak/poised enhancer and active promoter as the chromatin states most affected by $C H D 8$ suppression.

To further dissect these results, we compared the number of peaks for each histone mark in controls and CHD8 knock-downs within each chromatin state. While differences between biological replicates did not support a statistically significant variation at enhancer and promoter states (Supp. Fig. 3), the number of peaks decorated by histone H3K36me3 at transcriptional elongation genomic regions was significantly lower in Sh2-Sh4 CHD8 (p value $<0.05$, t test) compared to controls (Fig. 2B). Within the transcriptional elongation regions, H3K36me3 reduction affected $\sim 50 \%$ of all expressed genes in human neuronal progenitors (out of protein coding genes with > 2 TPM: 5447 lose H3K36me3, while 6451 were not affected). Additionally, genes that lost H3K36me3 following CHD8-haploinsufficiency, are significantly longer (90290 
vs $60902 \mathrm{bp}$ ) and composed of larger exon number (7.36 vs 6.20) (not shown), compared to the unaffected ones. Importantly, independent western blot quantification of total histone H3K36me3 levels in the three Sh1-Sh2-Sh4 CHD8 knock-down clones confirmed reduction of this histone modification compared to control Sh-GFP (Fig. 2 C-D). Genes with reduced H3K36me3 following CHD8 suppression at transcriptional elongation sites were strongly enriched for processes related to cell cycle and proliferation ("cell cycle", cell cycle process", "mitotic cell cycle", " mitotic cell cycle process") and "cell response to DNA damage stimulus" biological processes (Fig. 2E). For instance, the HCN1, gene encoding for a hyperpolarization gated chloride channel involved in epilepsy (Nava et al. 2014; Marini et al. 2018), presents reduced H3K36me3 enrichment following CHD8 suppression, while other actively transcribed genomic locations (i.g. BIRC6) showed unaltered distribution (Suppl. Fig.4).

CHD8 suppression-dependent reduction in Histone H3 Lysine 36 trimethylation impacts on CHD8-bound genes.

By overlaying human CHD8 binding sites on the previously established chromatin states (Fig. 2A), we confirmed that CHD8 was confined to active promoters $(90.11 \%)$ and, less prominently, to enhancers (strong, weak/poised a and b (3.18\%, 3.00\%, and 1.34\%)) (Suppl. Fig. 5A) (Sugathan et al. 2014). As previously reported (Wade et al. 2018), CHD8 binding correlated with higher histone H3K36me3 (Suppl. Fig. 5B-E) and H3K4me3 enrichment metagene profiles (Suppl. Fig. 5C-F), as well as elevated RNA expression levels compared to CHD8-unbound genes (Suppl. Fig. 5 D). 
Upon CHD8 suppression, stringently defined (see materials and methods) CHD8-bound genes appeared to be more sensitive than CHD8-unbound genes (988 and 4205 respectively), presenting a significantly reduced $\mathrm{H} 3 \mathrm{~K} 36 \mathrm{me} 3$ enrichment profile as confirmed by the effect size analysis (Fig. 3A-B. Suppl. Fig. 6A). Notably, the reduction in histone H3K36me3 elicited by CHD8 suppression was specific since histone $\mathrm{H} 3 \mathrm{~K} 4 \mathrm{me} 3$, another histone modification enriched at transcriptional start sites (TSS) of highly expressed genes (Suppl. Fig. 7A-B), remained unaltered (Suppl. Fig. 7C-E).

Remarkably, clustering analysis based on CHD8 binding sites enrichment in control hiNPC identified 3 different clusters: Cluster \#1 composed of 1239 genes with high CHD8 enrichment $($ mean $\log 2(\mathrm{ChIP} / \mathrm{INPUT})=0.27)$, cluster \#2 composed of 2429 genes with mediumlow CHD8 enrichment (mean $\log 2(\mathrm{ChIP} / \mathrm{INPUT})=-0.04)$ and cluster \#3 composed of 1380 genes with negligible CHD8 enrichment (mean $\log 2($ ChIP/INPUT) $=-0.31)$ (Fig. 3C-D). Strikingly, clusters \#1 was strongly affected by CHD8 decline, displaying significantly reduced H3K36me3 levels across the gene body (Fig. 3E and Suppl. Fig. 6B). Cluster \#2 and \#3, instead, with poor CHD8 enrichment in control hiNPC, displayed a correspondingly lower H3K36me3 enrichment, with no significant difference following CHD8 suppression (Fig. 3F-G and Suppl. Fig. 6C-D). In conclusion, this analysis confirmed that CHD8-bound genes were strongly sensitive to CHD8 reduction, presenting a substantial and specific drop in H3K36me3 histone modification (Fig. 3 C-E, Suppl. Fig.6C-D). Functional enrichment of genes from Clusters \#1, i.e. CHD8-bound and losing H3K36me3 enrichment upon CHD8-suppression, highlighted GO Biological Process terms related to 'mRNA processing' and 'RNA splicing' (Suppl. Fig. 8 and Suppl. Table 1). 
Histone H3 Lysine 36 trimethylation elicited by CHD8 suppression alters RNA alternative splicing.

To gauge the functional significance of H3K36me3 reduction observed following CHD 8 suppression, we then leveraged RNA-seq data from controls and CHD8-knockdown clones. As histone H3K36me3 seems to be correlated with high levels of RNA expression (suppl. Fig. 7A) (Wagner and Carpenter 2012), we reasoned that a decline in H3K36me3 levels would be associated with reduced RNA expression levels. Unexpectedly, however, haploinsufficient levels of CHD8 and, impaired H3K36me3 enrichment, didn't correspond to a global difference in transcription in either CHD8-bound or CHD8-unbound genes (Fig. 4A-B).

Importantly, a significant proportion of genes losing H3K36me3 upon CHD8-suppression (905 genes of which 326 (36\%) with CHD8 binding sites in promoter or enhancer regions) presented altered alternative splicing profiles, as evidenced by two analysis approaches (Fig. 4CD and Suppl. Fig. 9) (Trincado et al. 2018; Shen et al. 2014). We noted an almost equal distribution of the H3K36me3 peaks lost following CHD 8 suppression between exonic and intronic regions (data not shown). While a significant correlation between reduction in H3K36me3 and alternative splicing was observed, however, the direct comparison of H3K36me3 lost peaks and differential splicing genomic coordinates revealed poor intersection. The vast majority of alternative splicing events with associated reduced H3K36me3 profiles were categorized as 'alternative first exon' (1892 (46.60\%)) or 'exon skipping' (759 (18.69\%)) (Fig. 4E,F and G). For the roughly 2000 differential splicing events detected by SUPPA (Fig. 4C,D), the proportion of genes presenting positive or negative $\triangle \mathrm{PSI}$ ( $\triangle \mathrm{PSI}=\mathrm{PSI}$ ctrl - PSI_KD) (positive values: exon inclusion, negative values: exons skipping) remained pretty similar, however, for 'exon skipping' and 'alternative 5' events' types (SE and A5), the majority (60\%) 
presented a negative $\triangle \mathrm{PSI}$, suggesting that reduction of $\mathrm{H} 3 \mathrm{~K} 36 \mathrm{me} 3$ could correlate with reduced exon inclusion/increased exon skipping. Among genes characterized by a reduction in H3K36me3 and presenting an altered splicing pattern following CHD8 suppression, overrepresentation of GO terms and pathways related to "histone modification", "covalent chromatin modification" and "mitotic cell cycle phase transition" (Suppl. Table 1) was observed. 


\section{Discussion}

Disruption of CHD8 from de novo protein truncating variants and structural variants is well established as a highly penetrant risk factor for ASD (Neale et al. 2012; Satterstrom et al. 2020); O'Roak et al. 2012a; Talkowski et al. 2012). Chromodomain helicase DNA-binding protein 8 (CHD8), initially described as interacting with $\beta$-catenin as negative regulator of WNT signaling (Sakamoto et al. 2000; Nishiyama et al. 2004; Nishiyama et al. 2009; Nishiyama et al. 2012), has important roles during nervous system development. Mice completely lacking Chd8 exhibit early embryonic lethality (between E5.5 and E7.5) (Nishiyama et al. 2009), while Chd8 hypomorphic mutations are associated with perinatal mortality, pronounced brain hyperplasia in surviving animals and altered transcriptional expression (Hurley et al. 2018; Suetterlin et al. 2018). CHD8, a member of the chromo-helicases, has the ability to directly bind DNA (Sugathan et al. 2014; Cotney et al. 2015) and to slide nucleosomes (Manning and Yusufzai 2017). ChIPsequencing has revealed 7000 CHD8 binding sites located at H3K27ac and H3K4me3 enriched regions, highly transcriptionally active promoters and enhancers (Sugathan et al. 2014; Cotney et al. 2015). However, with both direct and indirect transcriptional effects observed following CHD8 suppression, its molecular mode of action in ASD remains unclear (Sugathan et al. 2014). CHD8 recruits histone KMT2/MLL methyltransferase complexes to induce mono-, di-, and trimethylation at lysine 4 of histone $\mathrm{H} 3$ and directly interacts with KMT2 components ASH2L and WDR5 (Thompson et al. 2008; Zhao et al. 2018). Reduced H3K27me3 enrichment was observed following CHD8 suppression, which might reflect alteration to the core PRC2 methyltransferase, Ezh2 (Durak et al. 2016). However, a myriad of functions of CHD8 complicates this interpretation as it is associated with elongating RNAPII, so its role in 
transcriptional elongation has to be considered, especially for highly expressed genes, that are densely decorated by histone H3K36me3 (Rodriguez-Paredes et al. 2009).

In our ASD-relevant, human neuronal progenitor model system, we observed that $C H D 8$ suppression is prominently associated with a depletion rather than a gain, in H3K4me1, $\mathrm{H} 3 \mathrm{~K} 27 \mathrm{ac}$ and H3K36me3 histone modifications. Genes displaying reduction in these histones marks are involved in 'Ribosome Biogenesis', 'Mitotic cell Division', 'Methyltransferase Activity' and strongly enriched for 'Essential, Intolerant to LoF genes', whose expression peaks early during nervous system development (M2, M3, post conception week 10-12) or increases during early cortical development (M16, post conception week 25-26) (Parikshak et al. 2013). The observed reduction in histone marks, while appreciable in all cases, is subtle and may suggest that CHD8 is unlikely to be a core component or a crucial recruiting factor for KMT2/COMPASS complex or SETD2/SETD5 methyltransferases. Rather it might act as a facilitator of histone methyltransferase activity. Using a combinatorial analysis of histone marks enrichment (Ernst and Kellis 2012), we dissected the differences elicited by CHD8 suppression into 10 chromatin states. Stringent statistical criteria to focus on the strongest and most reliable epigenetic changes supported a drastic depletion of H3K36me3 peaks in the transcription elongation chromatin state. While an effect at enhancers and promoters could not be completely ruled out, CHD8 seems to facilitate the methyltransferase activity leading to H3K36 trimethylation. Given the dynamic balance between the activity of histone methyltransferases and demethylases, we also cannot exclude the hypothesis that CHD8 might work as an inhibitor of KDM2B (He et al. 2013). GO terms associated with 'cell cycle progression' and 'mitosis' are enriched among genes losing $\mathrm{H} 3 \mathrm{~K} 36 \mathrm{me} 3$ at transcriptional elongation states. Interestingly, SETD2-5/KDM2B dysregulation has been correlated to the change of cell-cycle regulators 
(Kawakami et al. 2015), with altered H3K36 trimethylation associated with aberrant cell proliferation and neoplastic transformations (Wang et al. 2007; Jaffe et al. 2013; Simon et al. 2014). However, the modulation of H3K36 methylation is connected with the cell cycle not only in the context of tumorigenicity, but also during neuronal development and differentiation. Mutations in SETD2 have been described in Sotos syndrome, childhood overgrowth condition with macrocephaly (Luscan et al. 2014) and in an ASD proband, also presenting macrocephaly (O'Roak et al. 2012a; Lumish et al. 2015), while disruptive mutations in SETD5 - a newly described H3K36me3 methyltransferase (Sessa et al. 2019) - are associated with ID/ASD (Kuechler et al. 2015; Rauch et al., 2012; De Rubeis et al. 2014; Szczaluba et al. 2016; Parenti et al. 2017; Rawlins et al. 2017) and 3p25.3 microdeletion syndrome (Kellogg et al. 2013). Thus, it is possible that $C H D 8$ suppression, through its ability to modulate H3K36me3 levels, might lead to aberrant cell cycle regulation and macrocephaly as observed in animal models and in CHD8autistic subjects (Bernier et al. 2014; Suetterlin et al. 2018). CHD8 direct targets, transcriptionally upregulated upon $C H D 8$ suppression (i.e. PML, HDAC7, CDK6 (Sugathan et al. 2014)) and implicated in cell cycle progression were indeed significantly enriched (Fisher's exact test $\mathrm{p}$ value: $1.13 \times 10^{-7}$ ) among genes that lose $\mathrm{H} 3 \mathrm{~K} 36 \mathrm{me}$, suggesting a functional interplay between cell-cycle, CHD8 and H3K36me3.

But how can CHD8 modulate H3K36me3? From RNA sequencing data, CHD8 suppression doesn't seem to correlate with a direct reduction in SETD2/SETD5 levels nor to upregulation in H3K36me3 KDM2B methyltransferase (not shown). Based on our data, genes bound at their promoters/enhancers by CHD8 specifically present a significant depletion in H3K36me3, thus suggesting a possible direct interplay between the chromodomain and the H3K36 tri-methylases. Indeed, SETD2 and CHD8 display similar temporal expression patterns 
during human brain development (data from BrainSpan Atlas) (Bernier et al. 2014), though direct binding between CHD8 and SETD2 or SETD5 still needs to be demonstrated. The H3K36me3 depletion, observed upon CHD8 suppression, is reminiscent of the function of another member of the CHD family, CHD1, which remodels nucleosomes within the gene body of actively transcribed gene (Petty and Pillus 2013). In fact, chdl loss alters H3K4me3 and H3K36me3 patterns throughout the yeast genome and loss of chdl causes increased cryptic transcription, altered splicing and nucleosome turnover within gene bodies (Petty and Pillus 2013; Lee et al. 2017). On the other hand, KIS-L in Drosophila is associated with all sites of transcriptionally active chromatin in a pattern that largely overlaps that of RNA Polymerase II (Pol II). Moreover, kismet mutant larvae, present severe reduction in the levels of elongating Pol II, suggesting that Kismet is also required for transcription elongation (Srinivasan et al. 2005). Similarly, it is tempting to hypothesize that CHD8, recruited to highly transcribed genes and in concert with SET2, might play crucial roles in nucleosome stability during elongation (Krogan et al. 2003) (Huang and Zhu 2018).

Trimethylation of $\mathrm{H} 3 \mathrm{~K} 36$ demarcates body regions of actively transcribed genes, providing signals for modulating transcription fidelity, mRNA splicing and DNA damage repair (Wagner and Carpenter 2012). Aberrant reduction in SETD2 and reduced levels of H3K36me3 in our data are not directly causative of transcriptional differences and this is coherent with previous findings (Simon et al. 2014). Rather, reduced H3K36me3 correlates with altered alternative splicing. This is likely linked to the kinetics of PolII progression, since, H3K36me3 decorated nucleosomes act as intrinsic pause sites for elongating RNAPII. Altered H3K36me3 enrichment, then, could alter splice site recognition and change exon inclusion (Wagner and Carpenter 2012). In any event, alternative splicing differences detected in this study as a 
consequence of $C H D 8$ suppression, are particularly relevant in the context of nervous system development and function, especially for genes correlated with "RNA splicing" and "mRNA processing". Interestingly, aberrant neuronal splicing has been previously related to Chd8 haploinsufficiency in a mouse model of ASD (Gompers et al. 2017). In this context, our work establishing, for the first time, a functional link between $\mathrm{H} 3 \mathrm{~K} 36 \mathrm{me} 3$ and $C H D 8$, provides a molecular mechanism that warrants further investigations especially in neurodevelopmental syndromes and ASD in particular.

Finally, elongation-coupled H3K36 methylation usually serves also as a docking site for the histone deacetylase complex Rpd3S, which restores the repressive chromatin environment following Pol II passage to prevent cryptic transcription initiation (Huang and Zhu 2018). According to this hypothesis, $C H D 8$ suppression-related $\mathrm{H} 3 \mathrm{~K} 36 \mathrm{me} 3$ reduction might also cause reduced repression in gene bodies, possibly resulting in increased uncontrolled, cryptic transcription. Although not possible to address in this study, which relied on previously generated poly-A plus RNA libraries, the analysis of spurious transcription as a consequence of H3K36me3 reduction, represents the next challenge for understanding chromatin-linked consequences in neurodevelopmental disorders and ASD in particular. 


\section{Methods}

\section{Cellular model}

Human iPSC-derived NPC line GM8330-8 (Sheridan et al. 2011), Sh1-CHD8, Sh2-CHD8, Sh4CHD 8 and Sh-GFP lines, previously generated by lentiviral delivery of shRNAs targeting CHD 8 and GFP coding sequences respectively (Sugathan et al. 2014), were kindly provided by Dr. Stephen Haggarty laboratory (Massachusetts General Hospital and Harvard Medical School, Boston, MA, U.S.A.).

Cells were cultured on poly-L-ornithine hydrobromide $(20 \mu \mathrm{g} / \mathrm{mL}$, Sigma $) /$ laminin $(3 \mu \mathrm{g} / \mathrm{mL}$, Life Technologies) - coated plates in hiNPC medium (70\% v/v DMEM (Life Technologies) completed with 30\% v/v HAM F12 (Euroclone), 2\% v/v B27 (Life Technologies), 1\% v/v Penicillin-Streptomycin solution (Life Technologies) and 1\% v/v L-Glutamine (Corning) and supplemented with EGF (20 ng/mL, Sigma), bFGF (20 ng/mL, R\&D) and Heparin $(5 \mu \mathrm{g} / \mathrm{mL}$, Sigma)). Semi-confluent monolayers of hiNPCs were maintained in $5 \% \mathrm{CO} 2,37^{\circ} \mathrm{C}$ humidified incubator.

\section{Protein extraction and western blot analysis}

hiNPC cells were washed with PBS and resuspended in $100 \mathrm{ml}$ of extraction buffer (10mM Hepes $\mathrm{pH}=8,10 \mathrm{mM} \mathrm{KCl}, 0.1 \mathrm{mM} \mathrm{MgCl} 2,0.1 \mathrm{mM}$ EDTA $\mathrm{pH}=8,0.1 \mathrm{mM}$ DTT and halt protease \& phosphatase inhibitor cocktail (Life Technology)). Samples were centrifuged at $5.000 \mathrm{rpm}$ for 10 minutes at $4^{\circ} \mathrm{C}$ to remove cytosolic fraction. Nuclear pellets were resuspended in $\mathrm{HCl} 0.2 \mathrm{~N}$ and put in rotation at $4{ }^{\circ} \mathrm{C}$ overnight. After centrifugation at $4.000 \mathrm{rpm}$ for 10 minutes at $4{ }^{\circ} \mathrm{C}$, supernatants containing nuclear proteins were recovered. Proteins were quantified by Bradford Protein Assay Kit (Sigma-Aldrich). Protein samples were separated by 4-12\% Bis-Tris Protein 
Gels (Thermo Fisher) and transferred on Amersham ${ }^{\mathrm{TM}}$ Protran $^{\mathrm{TM}} 0.45 \mu \mathrm{m}$ Nitrocellulose (GEhealthcare) membrane. Membranes were blocked with 5\% w/v nonfat dried milk and incubated with the following primary antibodies: anti-CHD8 (Novus Biologicals NB-10060417) (1:1000), anti-HSP90 (Cell Signaling Tech. \#4874S) (1:5000), anti-Histone H3 (1:1.000) (Cell Signaling Tech, \#4499), anti-Histone H3K36me3 (1:1.000) (Abcam \#ab9050). Proteins were detected using horseradish peroxidase conjugated secondary antibodies anti-rabbit IgG 1:7.500 (GeneTex, \#GTX213110-01), visualized by ECL Select WB detection reagent (GE Healthcare) following manufacturer's instructions. Signal quantification was performed with Imagelab software (BIORAD).

Statistical Analysis

The analysis of target proteins differences in western blotting experiments was evaluated by performing an unpaired, one-tailed t test. In all t tests, the significance level was set to 0.05 . Data were represented as mean \pm Standard Deviation $(\mathrm{SD})$. The significance level was reported as: not significant (NS) $\mathrm{p}>0.05, * \mathrm{p} \leq 0.05, * * \mathrm{p} \leq 0.01, * * * \mathrm{p} \leq 0.001$.

\section{Chromatin immunoprecipitation and sequencing (ChIP-seq)}

Chromatin immunoprecipitation was performed using the protocol described by (Bernstein et al. 2006); with minor modifications. Briefly, $\sim 25$ million iPSC-derived neural progenitor cells, controls and $C H D 8$-Shs were fixed with $1 \%$ formaldehyde incubated for 10 min at RT with rotation. The crosslinking was quenched by adding $1.1 \mathrm{ml} 2.5 \mathrm{M}$ Glycine, incubated 5 min at RT with rotation. The cells were pelleted at $1000 \mathrm{rpm}$ and resuspended ice-cold PBS/protease inhibitor (PI) and spin for 5 minutes at $1000 \mathrm{rpm}$, washed with ice-cold PBS twice, harvested, pelleted and directly resuspended in 300ul lysis buffer/PI (50 mM Tris-HCl (pH8.1), 1\% SDS, 
$10 \mathrm{mM}$ EDTA), kept on ice for 10 minutes rotating occasionally and vortexed vigorously for 15 seconds every 3 minutes. Sonication of the samples 200-700 bps smear was accomplished using a Bioruptor sonicator (Diagenode), for a total of 45 minutes sonication at full power and sonication cycles of 30"ON/30"OFF. Samples were centrifuged at max speed for 10 minutes at $4^{\circ} \mathrm{C}$. Then, sheared-chromatin was diluted 10 fold in ChIP dilution buffer $(16.7 \mathrm{mM}$ Tris- $\mathrm{HCl}$ (pH 8.1), $167 \mathrm{mM} \mathrm{NaCl}, 0.01 \%$ SDS, 1.1\% Triton X-100, $1.2 \mathrm{mM}$ EDTA), supplemented with protease inhibitor. $50 \mu \mathrm{l}$ of sheared chromatin was removed and stored at $4^{\circ} \mathrm{C}$ as control aliquot (INPUT). Each sample was incubated at $4^{\circ} \mathrm{C}$ overnight with antibodies (20 ug/ChIP) of interest. The following primary antibodies were used: H3K27me3 (07-449, Millipore), H3K4me3 (AB8580, Abcam), H3K36me3 (Ab9050, Abcam), H3K4me2 (AB7766, Abcam), H3K4me1 (AB8895, Abcam) and H3K27ac (AB4729, Abcam). Chromatin-Antibody complexes were precipitated with Dynabeads Protein A beads (Invitrogen) and washed sequentially with low-salt (20 mM Tris-HCl (pH 8.1), 150 mM NaCl, 0.1\% SDS, $1 \%$ Triton X-100, 2 mM EDTA), highsalt (20 mM Tris-HCl (pH 8.1), $500 \mathrm{mM} \mathrm{NaCl,} \mathrm{0.1 \% SDS,} \mathrm{1 \%} \mathrm{Triton} \mathrm{X-100,} 2$ mM EDTA), LiCl (10 mM Tris- $\mathrm{HCl}(\mathrm{pH} 8.1), 0.25 \mathrm{M} \mathrm{LiCl,} 1 \%$ NP40, 1\% sodium deoxycholate, $1 \mathrm{mM}$ EDTA), and TE wash buffers (10 mM Tris- $\mathrm{HCl}$ (pH 8.0), $1 \mathrm{mM}$ EDTA). Immunoprecipitated chromatin and INPUT samples were then eluted in elution buffer (TE plus 1\% SDS, $150 \mathrm{mM}$ $\mathrm{NaCl}, 5 \mathrm{mM}$ DTT), de-crosslinked at $65^{\circ} \mathrm{C}$ for overnight and treated with proteinase $\mathrm{K}$. DNA isolation was performed by phenol:chloroform:isoamyl alcohol. DNA was precipitated with 200 $\mathrm{mM} \mathrm{NaCl}$, supplemented with $30 \mu \mathrm{g}$ glycogen, washed with EtOH and then treated with RNase I (Invitrogen). Finally, DNA was purified with MinElute Kit (Qiagen). Quantification of ChIP and INPUT DNA was accomplished using Qubit 2.0 Fluorometer system (Invitrogen). ChIP-seq libraries were prepared using NEBNext UltraII DNA Library preparation kit (Illumina) 
following manufacturer's instruction with no modification. ChIP-seq libraries were prepared starting from 5ng of fragmented DNA using NEBNext UltraII DNA Library preparation kit (Illumina) following manufacturer's instruction with no modifications. In order to obtain enough material for sequencing, eight cycles of PCR amplification were performed on adaptor ligated fragments.

\section{Histone marks ChIP-seq Data Analysis}

ChIP-seq reads were aligned on the human genome reference assembly GRCh38 using BWA (version 0.7.15) ( $\mathrm{Li}$ and Durbin 2009). Aligned reads were filtered to discard unmapped, multiply mapped, PCR duplicates reads (Picard tools MarkDuplicates version: 2.3.0, Picard Toolkit.” 2019. Broad Institute, GitHub Repository. http://broadinstitute.github.io/picard/) along with low quality alignments (samtools view -q 1, samtools version 1.7) (Li et al. 2009). Peak calling was performed with MACS2 (version 2.1.0) using a minimum FDR threshold 0.00001. The same settings with the addition of the --broad option were used for H3K27me 3 and H3K36me3 marks (Zhang et al. 2008). Peaks localized to blacklisted regions (http://mitra.stanford.edu/kundaje/akundaje/release/blacklists/) or unplaced contigs were filtered. Narrow peaks (for H3K27ac, H3K4me1, H3K4me2, H3K4me3 histone marks) closer than 350 bp were merged into a single peak. Peaks were considered common between replicates if they overlapped by at least $50 \%$ of the length of the shortest peak, then extended coordinates were maintained and used in downstream analyses. CHD8 ChIP-seq reads from Sugathan et al. 2014 were realigned to the reference GRCh38 and peaks were recalled with the same procedure used for the narrow histone marks, with a default FDR threshold 0.05 as only peaks identified by all three antibodies were retained. GENCODE v.26 was used for peaks annotation (Frankish et al. 2019). Genes losing H3K4me1, H3K27ac and H3K36me3 peaks in CHD8 knock-down were 
tested for enrichment of GO terms with the enricher function from clusterProfiler (version 3.10.1, Yu et al. 2012) with Benjamini-Hochberg adjusted $\mathrm{p}$ value cutoff of 0.05 . The full list of slimGO terms used was downloaded from Ensembl BioMart on September $9^{\text {th }}, 2019$. Enrichment of the same gene list was assessed on custom gene sets by using one-tailed Fisher's exact test. Custom gene sets were derived from public databases and publications of interest (Supplementary Table 1). Only non-redundant gene lists enriched at p value $<0.01$ were shown in Fig. 1E, complete enrichment results are available in Supplementary Table 1. Combining the histone marks enrichment patterns over the genome, 10 chromatin states were identified for control (Sh-GFP, Sh-GFP2) using ChromHMM (version 1.14) (Ernst and Kellis 2012). The states were manually annotated according to the literature. The number of peaks called in these regions in both replicates for each histone mark was counted using BEDTools intersect, version 2.25.0 (Quinlan and Hall 2010), and the difference between control and CHD8 knock-down was calculated. The total number of peaks for each histone marks in percentage was plotted as heat map in control cells (Fig. 2A), the difference (Fig. 2B) refers to the percentage of control peaks CHD8 KD peaks. The number of peaks per mark called in each chromatin state was tested with a two-sided t test considering two replicates in both control and CHD 8 knock-down. To generate the metagene profiles with deepTools, version 3.2.1, (Ramirez et al. 2016), ChIP-seq samples were normalized to INPUT with the SES method (Diaz et al. 2012). Enrichment was calculated in $10 \mathrm{bp}$ bins over the gene body, scaled to $5 \mathrm{Kbp}$, and $2 \mathrm{Kbp}$ up- and down-stream of the gene. In order to minimize noise in the metagene profile plots, a stringent set of filters was applied to protein coding genes before plotting: a minimum length of $2 \mathrm{Kbp}$, a minimum distance of $4 \mathrm{Kbp}$ from other genes and absence of other features on the opposite strand, leading to a set of 9442 protein coding genes. For each histone mark, only genes with enrichment were plotted (at least 
one non-zero bin). These genes were divided into three groups based on CHD 8 binding enrichment pattern via k-means with deepTools plotProfile command. To complement the statistical hypothesis testing, paired Cohen's d effect size statistics were calculated between groups along the entire region (Gibbons 1993). 99\% simultaneous confidence intervals were constructed controlling FWER (Bonferroni correction). CHD8 binding sites profiles were calculated with deepTools computeMatrix reference-point and plotted with deepTools plotProfile. Visualization of enrichment tracks for chromatin was performed with the Integrative Genomic Viewer (IGV, version 2.4.9 (Robinson et al. 2011)).

\section{RNA-seq Data Analysis}

Raw reads obtained from Sugathan et al., 2014 for corresponding samples (Sh-GFP, Sh-GFP2, Sh2-CHD8, Sh4-CHD8) were used to calculate transcripts abundance by kallisto (version 0.44.0) (Bray et al. 2016) on GENCODE v.26 transcripts. Transcripts per Million (TPM) were used to plot expression levels. SUPPA (version 2.3) was used to calculate the percent spliced-in (PSI) value per splicing event with an empirical method (Trincado et al. 2018). The same raw reads from Sugathan et al. 2014 were aligned with STAR (version 2.6) (Dobin et al. 2013) with default parameters on the GRCh38 reference, to analyze the alternative splicing with rMATS (version 3.1.0, Shen et al. 2014). As the two alternative splicing analysis methods are complementary (https:/github.com/comprna/SUPPA/issues/47), both were included in the analysis. To check the overlap of splicing events with chromatin marks, the spliced in/out exon coordinates were intersected with the histone mark peaks' coordinates. Alternative splicing events were represented in sashimi plots via ggsashimi (version 0.4.0) (Garrido-Martin et al. 2018) using GENCODE annotation v.33 as a reference (Frankish et al. 2019). 
bioRxiv preprint doi: https://doi.org/10.1101/2020.03.14.992032. The copyright holder for this preprint (which was not peer-reviewed) is the author/funder. All rights reserved. No reuse allowed without permission. 


\section{Acknowledgments}

We are indebted to the Biagioli, Ferrari and Talkowski lab members for helpful discussions, to Dr. Albert Basson (King's College, UK) and Dr. Fulvio Chiacchiera (Department CIBIO, University of Trento) for critical reading of the manuscript. We are grateful to Dr. Alessandro Quattrone (Director, Department CIBIO, University of Trento) for the scientific support to CIBIO Core Facilities research. We thank Drs. Veronica De Sanctis and Roberto Bertorelli for technical support and assistance with preparation and sequencing of libraries. We thank Dr. Stephen Haggarty (Massachusetts General Hospital and Harvard Medical School, Boston, MA, U.S.A.) for sharing the human neuronal progenitor cell lines. This work was supported by Department CIBIO Institutional funding to M.B. and S.P, grants from Autism Speaks and the Simons Foundation Autism Research Initiative to J.F.G. and the National Institutes of Health GM061354 and NS093200 to J.F.G and M.E.T. E.S. was supported by the Structured International Post-doc Program of SEMM (SIPOD) and the AFM-TELETHON fellowship n. 21835.

Author contributions: E.K, T.T., S.E., E.Salviato, F.D.L., E.S., M.A., performed experiments and analyzed the data. E.Salviato assisted with statistical analysis. E.S. and M.Benelli assisted with data analysis of alternative splicing and histone modifications. E.K. and M.Biagioli designed the study and wrote the paper. J.F.G., S.P., F.D., F.F., and M.E.T. provided intellectual guidance and assistance with bioinformatic analyses. M. Biagioli, M.E.T. and F.F. supervised this study. All authors discussed the results and commented on the manuscript. 


\section{References}

Abrahams BS, Arking DE, Campbell DB, Mefford HC, Morrow EM, Weiss LA, Menashe I, Wadkins T, Banerjee-Basu S, Packer A. 2013. SFARI Gene 2.0: a community-driven knowledgebase for the autism spectrum disorders (ASDs). Molecular autism 4: 36.

Bernier R, Golzio C, Xiong B, Stessman HA, Coe BP, Penn O, Witherspoon K, Gerdts J, Baker C, Vulto-van Silfhout AT et al. 2014. Disruptive CHD8 mutations define a subtype of autism early in development. Cell 158: 263-276.

Bernstein BE, Mikkelsen TS, Xie X, Kamal M, Huebert DJ, Cuff J, Fry B, Meissner A, Wernig M, Plath K et al. 2006. A bivalent chromatin structure marks key developmental genes in embryonic stem cells. Cell 125: 315-326.

Bray NL, Pimentel H, Melsted P, Pachter L. 2016. Near-optimal probabilistic RNA-seq quantification. Nature biotechnology 34: 525-527.

Consortium EP. 2012. An integrated encyclopedia of DNA elements in the human genome. Nature 489: 57-74.

Cotney J, Muhle RA, Sanders SJ, Liu L, Willsey AJ, Niu W, Liu W, Klei L, Lei J, Yin J et al. 2015. The autism-associated chromatin modifier CHD8 regulates other autism risk genes during human neurodevelopment. Nat Commun 6: 6404.

Cuajungco MP, Leyne M, Mull J, Gill SP, Lu W, Zagzag D, Axelrod FB, Maayan C, Gusella JF, Slaugenhaupt SA. 2003. Tissue-specific reduction in splicing efficiency of IKBKAP due to the major mutation associated with familial dysautonomia. Am J Hum Genet 72: 749758. 
Darnell JC, Van Driesche SJ, Zhang C, Hung KY, Mele A, Fraser CE, Stone EF, Chen C, Fak JJ, Chi SW et al. 2011. FMRP stalls ribosomal translocation on mRNAs linked to synaptic function and autism. Cell 146: 247-261.

Davis CA, Hitz BC, Sloan CA, Chan ET, Davidson JM, Gabdank I, Hilton JA, Jain K, Baymuradov UK, Narayanan AK et al. 2018. The Encyclopedia of DNA elements (ENCODE): data portal update. Nucleic acids research 46: D794-D801.

De Rubeis S, He X, Goldberg AP, Poultney CS, Samocha K, Cicek AE, Kou Y, Liu L, Fromer M, Walker S et al. 2014. Synaptic, transcriptional and chromatin genes disrupted in autism. Nature 515: 209-215.

Diaz A, Park K, Lim DA, Song JS. 2012. Normalization, bias correction, and peak calling for ChIP-seq. Statistical applications in genetics and molecular biology 11: Article 9.

Dobin A, Davis CA, Schlesinger F, Drenkow J, Zaleski C, Jha S, Batut P, Chaisson M, Gingeras TR. 2013. STAR: ultrafast universal RNA-seq aligner. Bioinformatics 29: 15-21.

Durak O, Gao F, Kaeser-Woo YJ, Rueda R, Martorell AJ, Nott A, Liu CY, Watson LA, Tsai LH. 2016. Chd8 mediates cortical neurogenesis via transcriptional regulation of cell cycle and Wnt signaling. Nature neuroscience 19: 1477-1488.

Ernst J, Kellis M. 2012. ChromHMM: automating chromatin-state discovery and characterization. Nature methods 9: 215-216.

Frankish A, Diekhans M, Ferreira AM, Johnson R, Jungreis I, Loveland J, Mudge JM, Sisu C, Wright J, Armstrong J et al. 2019. GENCODE reference annotation for the human and mouse genomes. Nucleic acids research 47: D766-D773. 
Garrido-Martin D, Palumbo E, Guigo R, Breschi A. 2018. ggsashimi: Sashimi plot revised for browser- and annotation-independent splicing visualization. PLoS computational biology 14: e1006360.

Gibbons ED, Hedeker, D.R. Devis, J.M. 1993. Estimation of Effect Size From a Series of Experiments Involving Paired Comparisons. Journal of Educational Statistics 18: 271279.

Gompers AL, Su-Feher L, Ellegood J, Copping NA, Riyadh MA, Stradleigh TW, Pride MC, Schaffler MD, Wade AA, Catta-Preta R et al. 2017. Germline Chd8 haploinsufficiency alters brain development in mouse. Nature neuroscience 20: 1062-1073.

He J, Shen L, Wan M, Taranova O, Wu H, Zhang Y. 2013. Kdm2b maintains murine embryonic stem cell status by recruiting PRC1 complex to $\mathrm{CpG}$ islands of developmental genes. Nature cell biology 15: 373-384.

Huang C, Zhu B. 2018. Roles of H3K36-specific histone methyltransferases in transcription: antagonizing silencing and safeguarding transcription fidelity. Biophysics reports 4: 170177.

Hurley S, Mohan C, Suetterlin P, Ellegood J, Rudari F, Lerch JP, Fernandes C, Basson MA. 2018. Non-monotonic regulation of gene expression, neural progenitor fate and brain growth by the chromatin remodeller CHD8. bioRxiv: 469031.

Iossifov I, O'Roak BJ, Sanders SJ, Ronemus M, Krumm N, Levy D, Stessman HA, Witherspoon KT, Vives L, Patterson KE et al. 2014. The contribution of de novo coding mutations to autism spectrum disorder. Nature 515: 216-221. 
Jaffe JD, Wang Y, Chan HM, Zhang J, Huether R, Kryukov GV, Bhang HE, Taylor JE, Hu M, Englund NP et al. 2013. Global chromatin profiling reveals NSD2 mutations in pediatric acute lymphoblastic leukemia. Nature genetics 45: 1386-1391.

Katayama Y, Nishiyama M, Shoji H, Ohkawa Y, Kawamura A, Sato T, Suyama M, Takumi T, Miyakawa T, Nakayama KI. 2016. CHD8 haploinsufficiency results in autistic-like phenotypes in mice. Nature 537: 675-679.

Kawakami E, Tokunaga A, Ozawa M, Sakamoto R, Yoshida N. 2015. The histone demethylase Fbx111/Kdm2a plays an essential role in embryonic development by repressing cell-cycle regulators. Mechanisms of development 135: 31-42.

Kellogg G, Sum J, Wallerstein R. 2013. Deletion of 3p25.3 in a patient with intellectual disability and dysmorphic features with further definition of a critical region. American journal of medical genetics Part A 161A: 1405-1408.

Kizer KO, Phatnani HP, Shibata Y, Hall H, Greenleaf AL, Strahl BD. 2005. A novel domain in Set2 mediates RNA polymerase II interaction and couples histone H3 K36 methylation with transcript elongation. Molecular and cellular biology 25: 3305-3316.

Krishnan A, Zhang R, Yao V, Theesfeld CL, Wong AK, Tadych A, Volfovsky N, Packer A, Lash A, Troyanskaya OG. 2016. Genome-wide prediction and functional characterization of the genetic basis of autism spectrum disorder. Nature neuroscience 19: 1454-1462.

Krogan NJ, Kim M, Tong A, Golshani A, Cagney G, Canadien V, Richards DP, Beattie BK, Emili A, Boone C et al. 2003. Methylation of histone H3 by Set2 in Saccharomyces cerevisiae is linked to transcriptional elongation by RNA polymerase II. Molecular and cellular biology 23: 4207-4218. 
Kuechler A, Zink AM, Wieland T, Ludecke HJ, Cremer K, Salviati L, Magini P, Najafi K, Zweier C, Czeschik JC et al. 2015. Loss-of-function variants of SETD5 cause intellectual disability and the core phenotype of microdeletion 3p25.3 syndrome. European journal of human genetics : EJHG 23: 753-760.

Lee Y, Park D, Iyer VR. 2017. The ATP-dependent chromatin remodeler Chd1 is recruited by transcription elongation factors and maintains $\mathrm{H} 3 \mathrm{~K} 4 \mathrm{me} 3 / \mathrm{H} 3 \mathrm{~K} 36 \mathrm{me} 3$ domains at actively transcribed and spliced genes. Nucleic acids research 45: 7180-7190.

Li H, Durbin R. 2009. Fast and accurate short read alignment with Burrows-Wheeler transform. Bioinformatics 25: 1754-1760.

Li H, Handsaker B, Wysoker A, Fennell T, Ruan J, Homer N, Marth G, Abecasis G, Durbin R, Genome Project Data Processing S. 2009. The Sequence Alignment/Map format and SAMtools. Bioinformatics 25: 2078-2079.

Luco RF, Misteli T. 2011. More than a splicing code: integrating the role of RNA, chromatin and non-coding RNA in alternative splicing regulation. Current opinion in genetics \& development 21: 366-372.

Luco RF, Pan Q, Tominaga K, Blencowe BJ, Pereira-Smith OM, Misteli T. 2010. Regulation of alternative splicing by histone modifications. Science 327: 996-1000.

Lumish HS, Wynn J, Devinsky O, Chung WK. 2015. Brief Report: SETD2 Mutation in a Child with Autism, Intellectual Disabilities and Epilepsy. Journal of autism and developmental disorders 45: 3764-3770.

Luscan A, Laurendeau I, Malan V, Francannet C, Odent S, Giuliano F, Lacombe D, Touraine R, Vidaud M, Pasmant E et al. 2014. Mutations in SETD2 cause a novel overgrowth condition. Journal of medical genetics 51: 512-517. 
Manning BJ, Yusufzai T. 2017. The ATP-dependent chromatin remodeling enzymes CHD6, CHD7, and CHD8 exhibit distinct nucleosome binding and remodeling activities. The Journal of biological chemistry 292: 11927-11936.

Marini C, Porro A, Rastetter A, Dalle C, Rivolta I, Bauer D, Oegema R, Nava C, Parrini E, Mei D et al. 2018. HCN1 mutation spectrum: from neonatal epileptic encephalopathy to benign generalized epilepsy and beyond. Brain : a journal of neurology 141: 3160-3178.

Mordes D, Luo X, Kar A, Kuo D, Xu L, Fushimi K, Yu G, Sternberg P, Jr., Wu JY. 2006. PremRNA splicing and retinitis pigmentosa. Mol Vis 12: 1259-1271.

Nava C, Dalle C, Rastetter A, Striano P, de Kovel CG, Nabbout R, Cances C, Ville D, Brilstra EH, Gobbi G et al. 2014. De novo mutations in HCN1 cause early infantile epileptic encephalopathy. Nature genetics 46: 640-645.

Neale BM, Kou Y, Liu L, Ma'ayan A, Samocha KE, Sabo A, Lin CF, Stevens C, Wang LS, Makarov V et al. 2012. Patterns and rates of exonic de novo mutations in autism spectrum disorders. Nature 485: 242-245.

Nilsen TW, Graveley BR. 2010. Expansion of the eukaryotic proteome by alternative splicing. Nature 463: 457-463.

Nishiyama M, Nakayama K, Tsunematsu R, Tsukiyama T, Kikuchi A, Nakayama KI. 2004. Early embryonic death in mice lacking the beta-catenin-binding protein Duplin. Molecular and cellular biology 24: 8386-8394.

Nishiyama M, Oshikawa K, Tsukada Y, Nakagawa T, Iemura S, Natsume T, Fan Y, Kikuchi A, Skoultchi AI, Nakayama KI. 2009. CHD8 suppresses p53-mediated apoptosis through histone H1 recruitment during early embryogenesis. Nature cell biology 11: 172-182. 
Nishiyama M, Skoultchi AI, Nakayama KI. 2012. Histone H1 recruitment by CHD8 is essential for suppression of the Wnt-beta-catenin signaling pathway. Molecular and cellular biology 32: 501-512.

O'Roak BJ, Vives L, Fu W, Egertson JD, Stanaway IB, Phelps IG, Carvill G, Kumar A, Lee C, Ankenman K et al. 2012a. Multiplex targeted sequencing identifies recurrently mutated genes in autism spectrum disorders. Science 338: 1619-1622.

O'Roak BJ, Vives L, Girirajan S, Karakoc E, Krumm N, Coe BP, Levy R, Ko A, Lee C, Smith JD et al. 2012b. Sporadic autism exomes reveal a highly interconnected protein network of de novo mutations. Nature 485: 246-250.

Parenti I, Teresa-Rodrigo ME, Pozojevic J, Ruiz Gil S, Bader I, Braunholz D, Bramswig NC, Gervasini C, Larizza L, Pfeiffer L et al. 2017. Mutations in chromatin regulators functionally link Cornelia de Lange syndrome and clinically overlapping phenotypes. Human genetics 136: 307-320.

Parikshak NN, Luo R, Zhang A, Won H, Lowe JK, Chandran V, Horvath S, Geschwind DH. 2013. Integrative functional genomic analyses implicate specific molecular pathways and circuits in autism. Cell 155: 1008-1021.

Petty E, Pillus L. 2013. Balancing chromatin remodeling and histone modifications in transcription. Trends in genetics : TIG 29: 621-629.

Quinlan AR, Hall IM. 2010. BEDTools: a flexible suite of utilities for comparing genomic features. Bioinformatics 26: 841-842.

Radman-Livaja M, Quan TK, Valenzuela L, Armstrong JA, van Welsem T, Kim T, Lee LJ, Buratowski S, van Leeuwen F, Rando OJ et al. 2012. A key role for Chd1 in histone H3 dynamics at the 3 ' ends of long genes in yeast. PLoS Genet 8: e1002811. 
Ramirez F, Ryan DP, Gruning B, Bhardwaj V, Kilpert F, Richter AS, Heyne S, Dundar F, Manke T. 2016. deepTools2: a next generation web server for deep-sequencing data analysis. Nucleic acids research 44: W160-165.

Rawlins LE, Stals KL, Eason JD, Turnpenny PD. 2017. De novo SETD5 nonsense mutation associated with diaphragmatic hernia and severe cerebral cortical dysplasia. Clinical dysmorphology 26: 95-97.

Robinson JT, Thorvaldsdottir H, Winckler W, Guttman M, Lander ES, Getz G, Mesirov JP. 2011. Integrative genomics viewer. Nature biotechnology 29: 24-26.

Rodriguez-Paredes M, Ceballos-Chavez M, Esteller M, Garcia-Dominguez M, Reyes JC. 2009. The chromatin remodeling factor CHD8 interacts with elongating RNA polymerase II and controls expression of the cyclin E2 gene. Nucleic acids research 37: 2449-2460.

Sakamoto I, Kishida S, Fukui A, Kishida M, Yamamoto H, Hino S, Michiue T, Takada S, Asashima M, Kikuchi A. 2000. A novel beta-catenin-binding protein inhibits betacatenin-dependent Tcf activation and axis formation. The Journal of biological chemistry 275: $32871-32878$.

Satterstrom FK, Kosmicki JA, Wang J, Breen MS, De Rubeis S, An JY, Peng M, Collins R, Grove J, Klei L et al. 2020. Large-Scale Exome Sequencing Study Implicates Both Developmental and Functional Changes in the Neurobiology of Autism. Cell 180: 568584 e523.

Selth LA, Sigurdsson S, Svejstrup JQ. 2010. Transcript Elongation by RNA Polymerase II. Annu Rev Biochem 79: 271-293.

Sessa A, Fagnocchi L, Mastrototaro G, Massimino L, Zaghi M, Indrigo M, Cattaneo S, Martini D, Gabellini C, Pucci C et al. 2019. SETD5 Regulates Chromatin Methylation State and 
Preserves Global Transcriptional Fidelity during Brain Development and Neuronal Wiring. Neuron 104: 271-289 e213.

Shen S, Park JW, Lu ZX, Lin L, Henry MD, Wu YN, Zhou Q, Xing Y. 2014. rMATS: robust and flexible detection of differential alternative splicing from replicate RNA-Seq data. Proc Natl Acad Sci U S A 111: E5593-5601.

Sheridan SD, Theriault KM, Reis SA, Zhou F, Madison JM, Daheron L, Loring JF, Haggarty SJ. 2011. Epigenetic characterization of the FMR1 gene and aberrant neurodevelopment in human induced pluripotent stem cell models of fragile X syndrome. PloS one 6: e26203.

Simon JM, Hacker KE, Singh D, Brannon AR, Parker JS, Weiser M, Ho TH, Kuan PF, Jonasch E, Furey TS et al. 2014. Variation in chromatin accessibility in human kidney cancer links H3K36 methyltransferase loss with widespread RNA processing defects. Genome research 24: 241-250.

Smolle M, Venkatesh S, Gogol MM, Li H, Zhang Y, Florens L, Washburn MP, Workman JL. 2012. Chromatin remodelers Isw1 and Chd1 maintain chromatin structure during transcription by preventing histone exchange. Nat Struct Mol Biol 19: 884-892.

Srinivasan S, Armstrong JA, Deuring R, Dahlsveen IK, McNeill H, Tamkun JW. 2005. The Drosophila trithorax group protein Kismet facilitates an early step in transcriptional elongation by RNA Polymerase II. Development 132: 1623-1635.

Suetterlin P, Hurley S, Mohan C, Riegman KLH, Pagani M, Caruso A, Ellegood J, Galbusera A, Crespo-Enriquez I, Michetti C et al. 2018. Altered Neocortical Gene Expression, Brain Overgrowth and Functional Over-Connectivity in Chd8 Haploinsufficient Mice. Cereb Cortex 28: 2192-2206. 
Sugathan A, Biagioli M, Golzio C, Erdin S, Blumenthal I, Manavalan P, Ragavendran A, Brand H, Lucente D, Miles J et al. 2014. CHD8 regulates neurodevelopmental pathways associated with autism spectrum disorder in neural progenitors. Proc Natl Acad Sci U S A 111: E4468-4477.

Szczaluba K, Brzezinska M, Kot J, Rydzanicz M, Walczak A, Stawinski P, Werner B, Ploski R. 2016. SETD5 loss-of-function mutation as a likely cause of a familial syndromic intellectual disability with variable phenotypic expression. American journal of medical genetics Part A 170: 2322-2327.

Talkowski ME, Rosenfeld JA, Blumenthal I, Pillalamarri V, Chiang C, Heilbut A, Ernst C, Hanscom C, Rossin E, Lindgren AM et al. 2012. Sequencing chromosomal abnormalities reveals neurodevelopmental loci that confer risk across diagnostic boundaries. Cell 149: $525-537$.

Thompson BA, Tremblay V, Lin G, Bochar DA. 2008. CHD8 is an ATP-dependent chromatin remodeling factor that regulates beta-catenin target genes. Molecular and cellular biology 28: 3894-3904.

Trincado JL, Entizne JC, Hysenaj G, Singh B, Skalic M, Elliott DJ, Eyras E. 2018. SUPPA2: fast, accurate, and uncertainty-aware differential splicing analysis across multiple conditions. Genome biology 19: 40.

Venkatesh S, Workman JL. 2015. Histone exchange, chromatin structure and the regulation of transcription. Nature reviews Molecular cell biology 16: 178-189.

Wade AA, Lim K, Catta-Preta R, Nord AS. 2018. Common CHD8 Genomic Targets Contrast With Model-Specific Transcriptional Impacts of CHD8 Haploinsufficiency. Frontiers in molecular neuroscience 11: 481. 
Wagner EJ, Carpenter PB. 2012. Understanding the language of Lys36 methylation at histone H3. Nature reviews Molecular cell biology 13: 115-126.

Wang ET, Cody NA, Jog S, Biancolella M, Wang TT, Treacy DJ, Luo S, Schroth GP, Housman DE, Reddy S et al. 2012. Transcriptome-wide regulation of pre-mRNA splicing and mRNA localization by muscleblind proteins. Cell 150: 710-724.

Wang GG, Cai L, Pasillas MP, Kamps MP. 2007. NUP98-NSD1 links H3K36 methylation to Hox-A gene activation and leukaemogenesis. Nature cell biology 9: 804-812.

Wang T, Birsoy K, Hughes NW, Krupczak KM, Post Y, Wei JJ, Lander ES, Sabatini DM. 2015. Identification and characterization of essential genes in the human genome. Science 350: 1096-1101.

Yasin H, Gibson WT, Langlois S, Stowe RM, Tsang ES, Lee L, Poon J, Tran G, Tyson C, Wong CK et al. 2019. A distinct neurodevelopmental syndrome with intellectual disability, autism spectrum disorder, characteristic facies, and macrocephaly is caused by defects in CHD8. Journal of human genetics 64: 271-280.

Yu G, Wang LG, Han Y, He QY. 2012. clusterProfiler: an R package for comparing biological themes among gene clusters. Omics : a journal of integrative biology 16: 284-287.

Zhang Y, Liu T, Meyer CA, Eeckhoute J, Johnson DS, Bernstein BE, Nusbaum C, Myers RM, Brown M, Li W et al. 2008. Model-based analysis of ChIP-Seq (MACS). Genome biology 9: R137.

Zhao C, Dong C, Frah M, Deng Y, Marie C, Zhang F, Xu L, Ma Z, Dong X, Lin Y et al. 2018. Dual Requirement of CHD8 for Chromatin Landscape Establishment and Histone Methyltransferase Recruitment to Promote CNS Myelination and Repair. Developmental cell 45: 753-768 e758. 


\section{Figures}

A

Human iPSC-derived NPCs

Control

Sh-GFP

Sh2-GFP

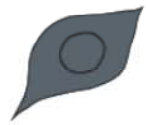

CHD8 knock down

Sh1-CHD8

Sh2-CHD8

Sh4-CHD8

(Sugathan et al., 2014)
Histone marks ChIP-seq

H3K4me2

$\mathrm{H} 3 \mathrm{~K} 4 \mathrm{me} 3$

Active promoters

H3K27me3 Inactive promoters

H3K4me1

H3K27ac

Enhancers

H3K36me3 Actively transcribed regions
Kerschbamer_Fig1

CHD8 ChIP-seq

(Sugathan et al., 2014)

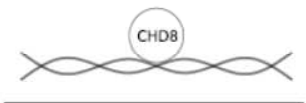

RNA-seq

(Sugathan et al., 2014)

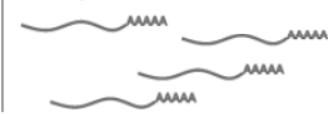

B

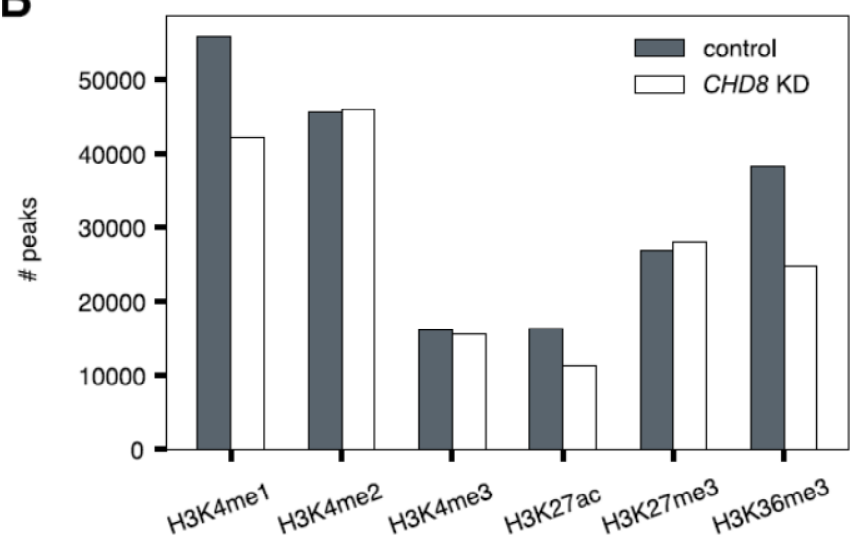

D

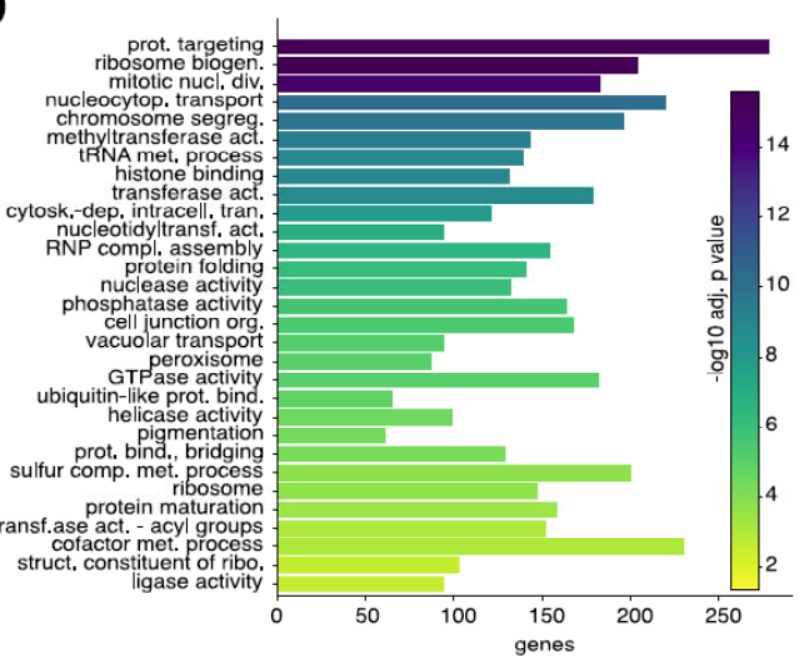

G

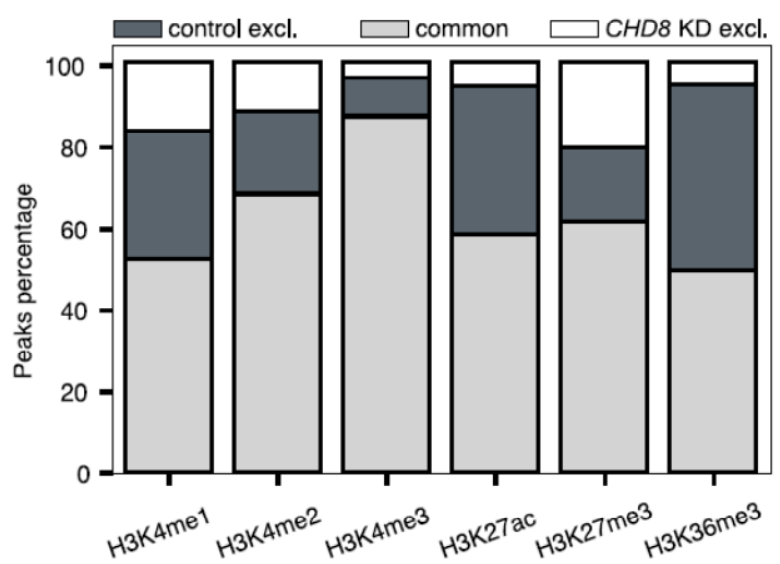

E

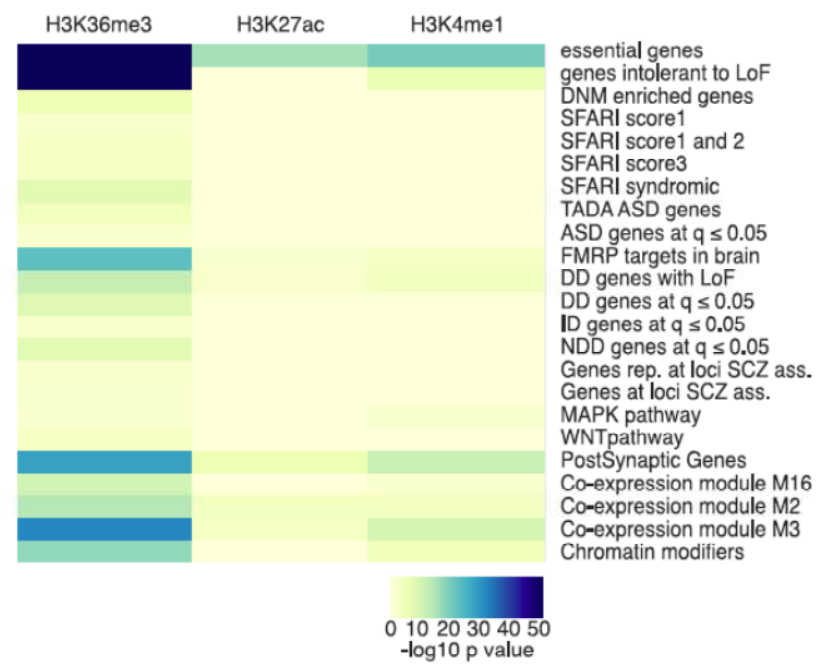

Figure 1 Altered chromatin landscape upon CHD8-suppression: reduction of histone H3K27ac, H3K36me 3 and H3K4mel. 
A. Schematic representation of the study design and integrative approach used in this work. Human iPSC-derived NPCs (hiNPC) knocked-down for CHD8 (Sh1-, Sh2- and Sh4-CHD8) and control hiNPCs (Sh-GFP, Sh-GFP2) (Sugathan et al. 2014), were analyzed via ChIP-seq for six histone marks representative of different chromatin regions - active promoters (H3K4me2, H3K4me3), inactive promoters (H3K27me3), enhancers (H3K4me1, H3K27ac) and actively transcribed regions (H3K36me3). ChIP-seq results were subsequently integrated with CHD8 binding sites and available transcriptomics (RNA-seq) datasets obtained from the same model system (Sugathan et al. 2014).

B. The bar plot reports the total number of peaks identified in controls (grey) and CHD8 knockdowns (white) for each histone mark analyzed. The y-axis presents the number of peaks resulting from the intersection of two replicates for each experimental condition: controls (intersection between Sh-GFP, Sh-GFP2), CHD8-knock-down (intersection between Sh2 and Sh4 CHD8).

Histone marks H3K4me1, H3K27ac and H3K36me3 were the mostly affected by CHD8 knockdown, displaying, in all cases, decreased number of peaks in CHD8 suppression hiNPC models (19388, 6445 and 20507 peaks were lost, respectively).

C. The bar plot highlights the percentage of peaks in common between controls (intersection between Sh-GFP, Sh-GFP2) and CHD8-knock-down (intersection between Sh2- and Sh4CHD8) (light gray). Percentage of peaks exclusively present in controls (intersection between Sh-GFP, Sh-GFP2) or in $C H D 8$ knock-down (intersection between Sh2- and Sh4-CHD8) are presented in grey and white, respectively. The total number of peaks $(100 \%)$ is the sum of the peaks common and exclusive to each experimental condition. Upon CHD8 suppression, H3K4me1, H3K27ac and H3K36me3 confirm extensive peak loss compared to controls, with a reduction of $1.22 \%, 36.23 \%$ and $45.20 \%$, respectively. 
D. Graphical representation of slim GO functional annotation enrichment for all genes interested by H3K4me1, H3K27ac and H3K36me3 peaks depletion following CHD8 knock-down. Top 30 GO terms are shown in figure, while full list of significant terms is reported in Suppl. Table 1. Color-coded bar plot according to adjusted $\mathrm{p}$ values in - $\log 10$ scale, displays statistically significant slim GOs. The size of the bar (x-axis) reports the number of genes for each slim GO term.

E. The heatmap represents gene set enrichment $\mathrm{p}$ values in $-\log 10$ scale for all genes losing H3K4me1, H3K27ac and H3K36me3 peaks following CHD8 knock-down. Genes' lists related to ASD, neurodevelopment, co-expression modules in brain and intolerance to loss of function, were tested for enrichment as described in Materials and Methods. Full gene list description and enrichment results are available in Supplementary Table 1. 
Kerschbamer_Fig2
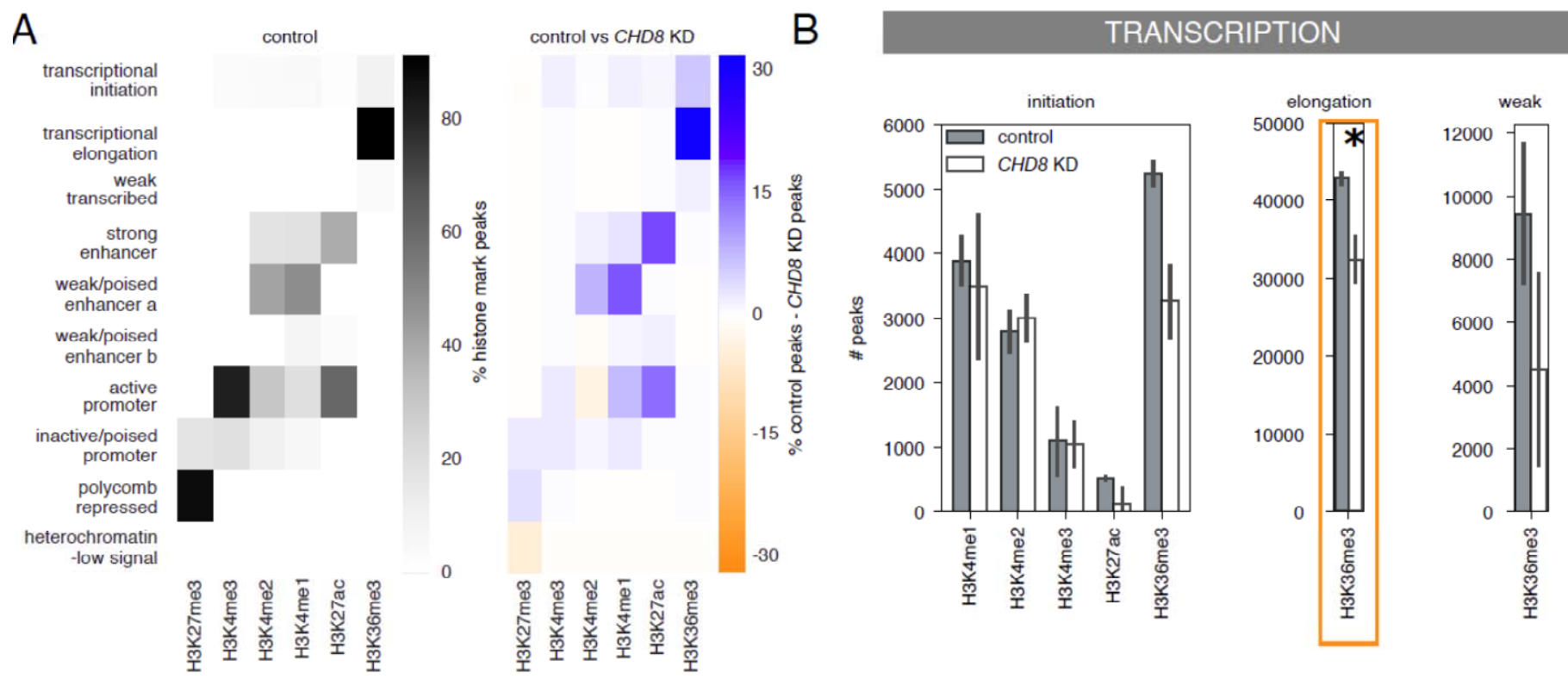

C

\section{$E$}

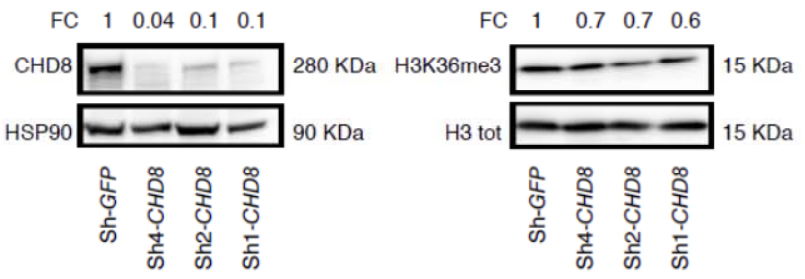

D
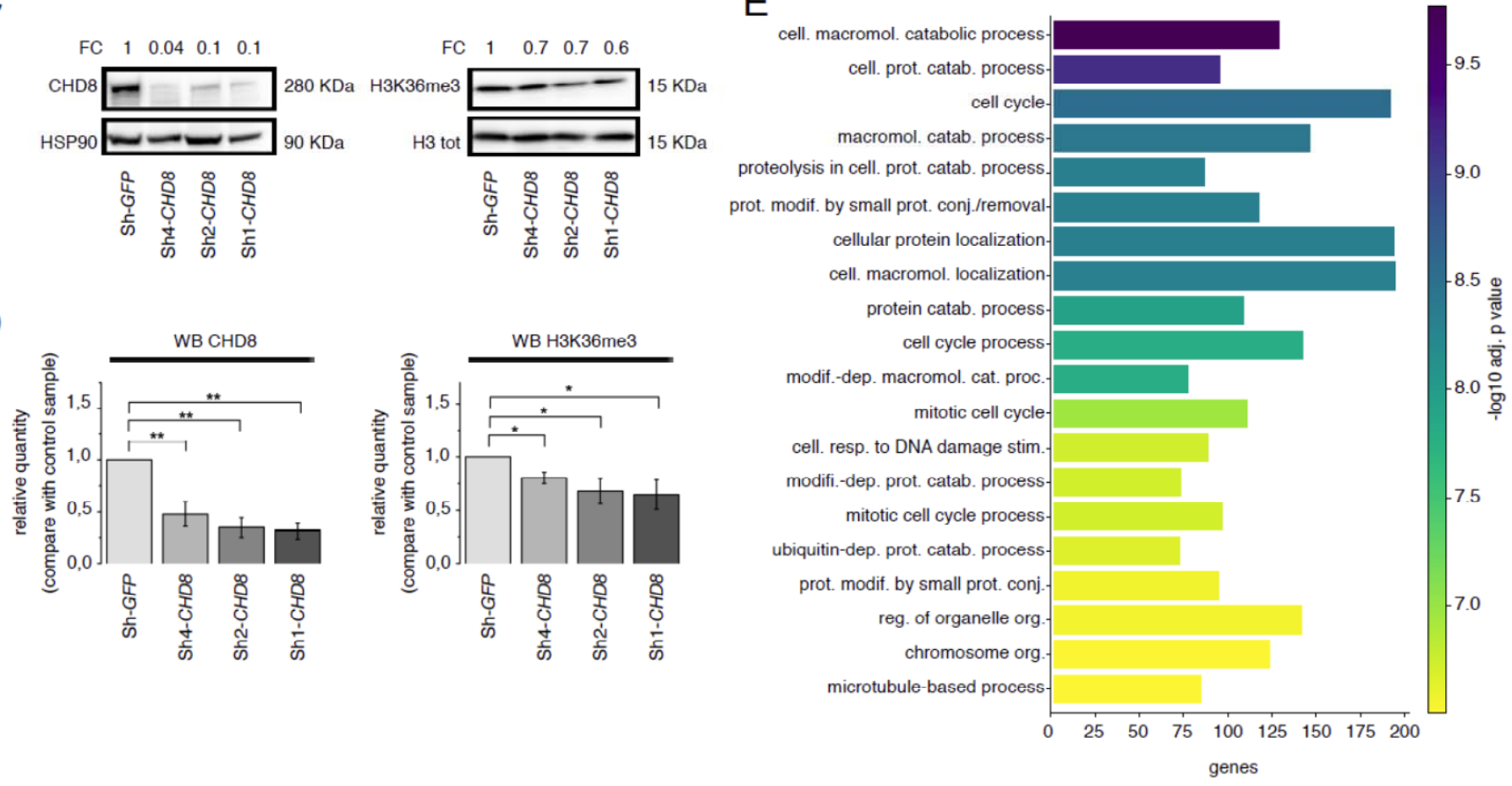

Figure 2 CHD8-suppression significantly impacts on histone H3K36me3 enrichment at transcriptional elongation sites.

A. The heatmaps represent 10 different chromatin states (1. transcriptional initiation, 2. transcriptional elongation, 3. weakly transcribed, 4. strong enhancer, 5. weak/poised enhancer a, 
6. weak/poised enhancer b, 7. active promoter, 8. inactive/poised promoter, 9. polycomb repressed, 10. heterochromatin/low signal), defined by the combination of different histone marks in control hiNPC as defined by ChromHMM(Ernst and Kellis 2012). The distribution of histone marks peaks across different chromatin states (see materials and methods for details) is presented as percentage of the total and color-coded in the heatmap (left).

On the right, the heatmap describes the difference in number of peaks between two experimental conditions (controls versus $C H D 8$ knock-down). In blue are indicated chromatin states enriched in control, in orange chromatin states enriched in CHD8 knock-down. H3K36me3 in transcriptional elongation is identified as the most affected chromatin state.

B. The bar plots represent the number of peaks for each histone mark identified at transcriptional initiation (left), elongation (center) and weakly transcribed (right) genomic regions. Grey bars indicate controls (n=2, Sh-GFP, Sh-GFP2) and white bars refer to CHD 8 knock-down (n=2, Sh2-CHD8, Sh4-CHD8). H3K36me3 peak loss upon CHD8 suppression was significant at the transcriptional elongation states (two biological replicates, T-test, $\mathrm{p}<0.05$ ).

C. The image describes a representative western blot illustrating CHD8 (left) and total histone H3K36me3 (right) levels across control (Sh-GFP) and CHD8 knock-down clones (Sh1-CHD8, Sh2-CHD8, Sh4-CHD8). Down-regulation of CHD8 and total H3K36me3 amount is observed following $C H D 8$ suppression. Levels of CHD8 and H3K36me3 reduction are indicated in the top part of the panel as fold change (FC) compared to control Sh-GFP. Comparable amounts of total protein were loaded and HSP90 and total histone H3 was used as loading control.

D. The bar chart reports fold change differences in CHD8 (left) and histone H3K36me3 left) levels comparing control (Sh-GFP) and CHD8 knock-down clones (Sh1-CHD8, Sh2-CHD8, Sh4-CHD8) in western blotting experiments. The bars represent normalized (HSP90 and total 
histone H3 were used as loading control) CHD8 and H3K36me3 values relative to Sh-GFP controls. Mean values \pm s.e. from independent biological replicates $(n=4$ for $\operatorname{Sh} 4-C H D 8$ and $n=6$ for the other samples) are plotted. $t$ test for two mean population was performed. $* \mathrm{p} \leq 0.05$.

E. Bar plot represents GO biological process terms significantly enriched in genes with a reduced level of H3K36me3 at transcriptional elongation sites upon CHD8 suppression. The bars are ordered according to adjusted $\mathrm{p}$ values in $-\log 10$ scale, the $\mathrm{x}$-axis represents the number of genes enriched for each term. Top $20 \mathrm{GO}$ terms are shown in figure, while full list of significant terms is reported in Suppl. Table 1. 
Kerschbamer_Fig3

A

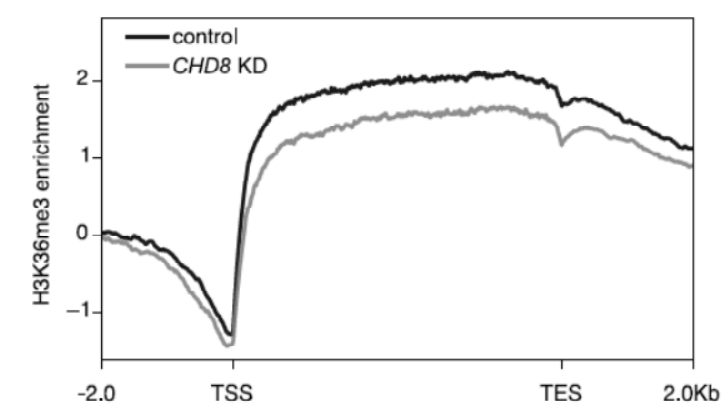

$\mathrm{C}$

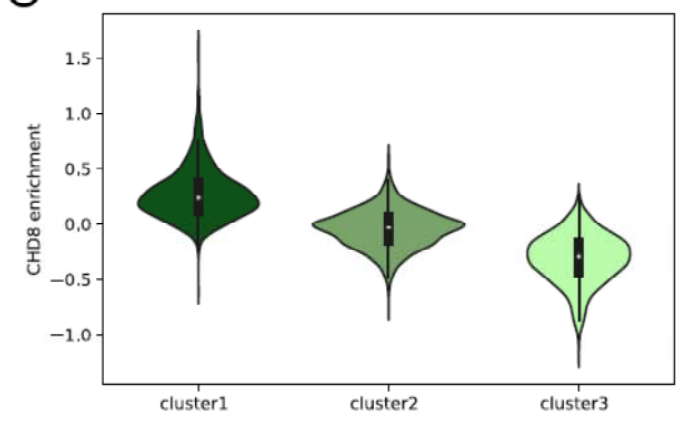

B

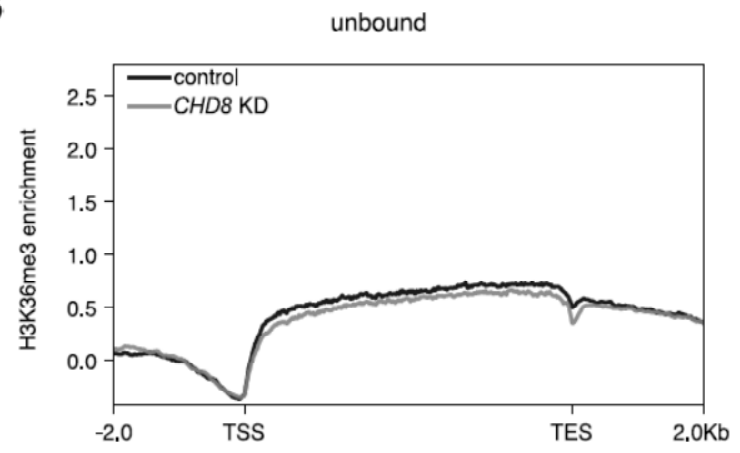

D

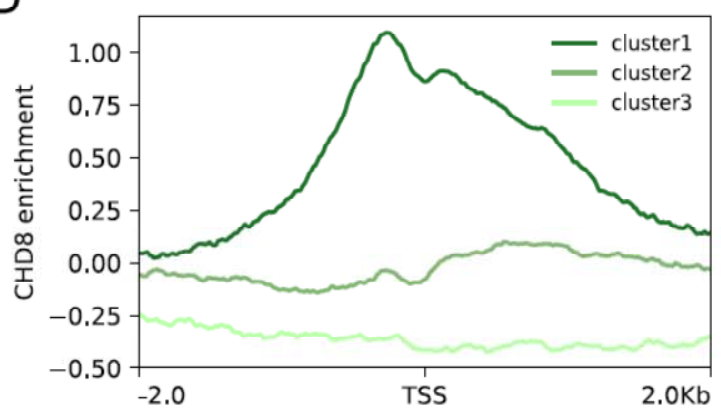

clustering by CHD8 binding
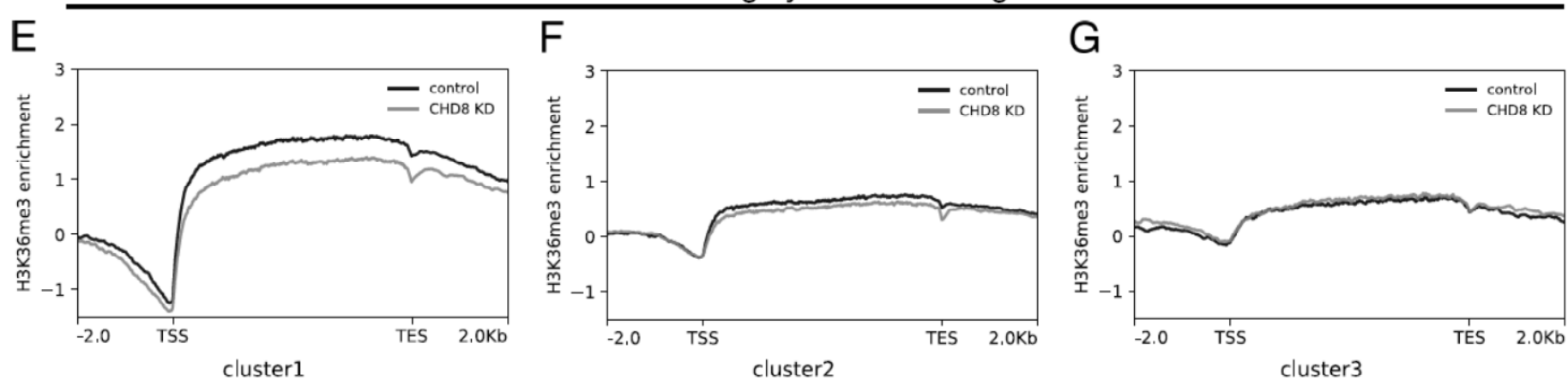

Figure 3 CHD8-suppression correlates with reduced H3K36me3 enrichment preferentially at CHD8-bound genes.

A. B. Metagene profiles display the average of histone H3K36me3 enrichment (scaled $\log 2$ ratio of normalized ChIP value over INPUT control - see also Materials and Methods) in a region of $\pm 2 \mathrm{Kbp}$ upstream the transcriptional start site (TSS) and downstream the transcriptional end site (TES), calculated for control (black line) and CHD8 knock-down (grey line) hiNPC and for 
CHD8-bound (\#988) (A) and CHD8-unbound genes (\#4205) (B). The difference between H3K36me3 enrichment in control and CHD8 knock-down is significant for CHD8-bound genes, but not for CHD8 unbound genes (paired Cohen's d effect size statistics).

C. The violin plots represent the level of CHD8 binding enrichment (log2(ChIP/INPUT) for 3 groups of genes as clustered by k-means. Cluster \#1 composed of 1239 genes shows high CHD8 enrichment $($ mean $\log 2($ ChIP/INPUT $)=0.27)$, cluster \#2 composed of 2429 genes with mediumlow CHD8 enrichment (mean $\log 2($ ChIP/INPUT) $=-0.04)$ and cluster \#3 composed of 1380 genes dispays negligible CHD8 enrichment (mean $\log 2($ ChIP/INPUT $)=-0.31)$.

D. Metagene profiles show the average of CHD8 binding enrichment in a region of $\pm 2 \mathrm{Kbp}$ around the TSS, calculated for the three clusters\#1, \#2, \#3 as described in C.

E. F. G. Metagene profiles display the average of histone H3K36me3 enrichment $(\log 2(\mathrm{ChIP} / \mathrm{INPUT}))$ in a region of $\pm 2 \mathrm{Kbp}$ upstream the TSS and downstream the TES, calculated for control (black line) and CHD 8 knock-down (grey line) hiNPC for each of the 3 clusters identified in Fig. 3C. The difference between H3K36me3 enrichment in control and CHD8 knock-down is significant for Cluster\#1, but not for Cluster\#2 or Cluster\#3 (paired Cohen's d effect size statistics, see suppl. Fig. 6B-D). 
A

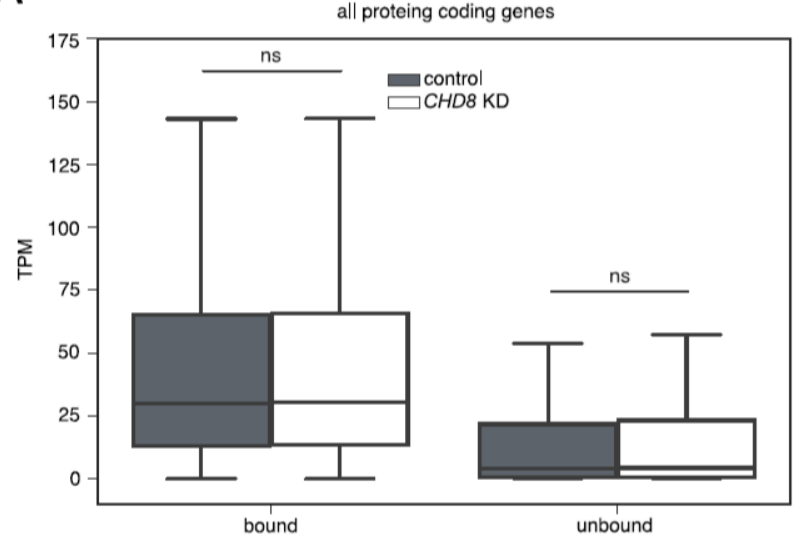

$\mathrm{C}$

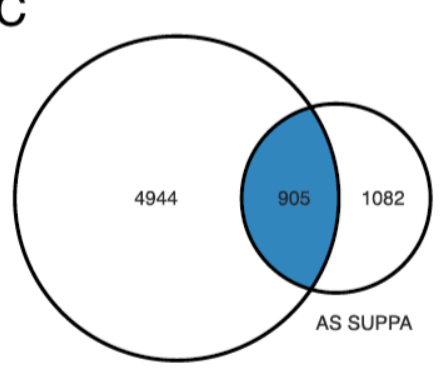

losing $\mathrm{H} 3 \mathrm{~K} 36 \mathrm{me} 3$ in $\mathrm{CHD} 8 \mathrm{KD}$ $\mathrm{p}=1.93 \mathrm{e}-57$
B

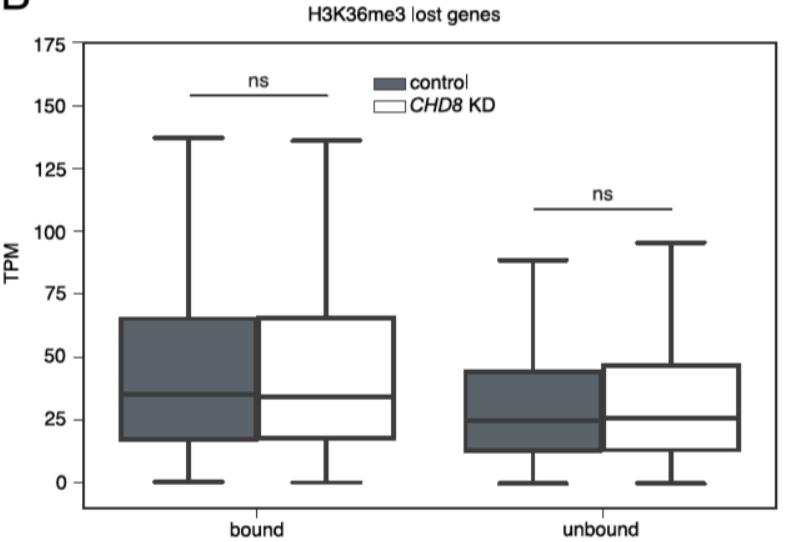

Kerschbamer_Fig4

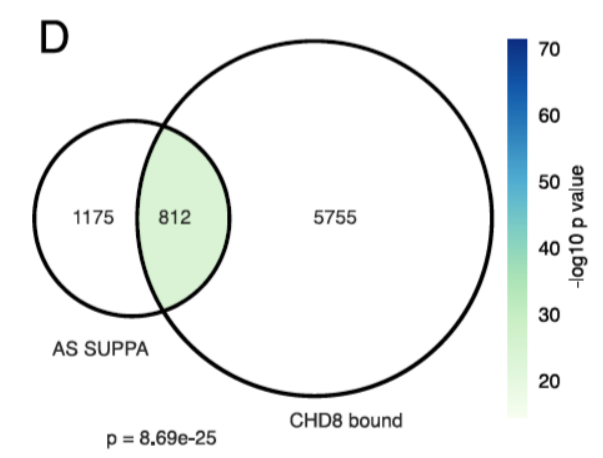

E
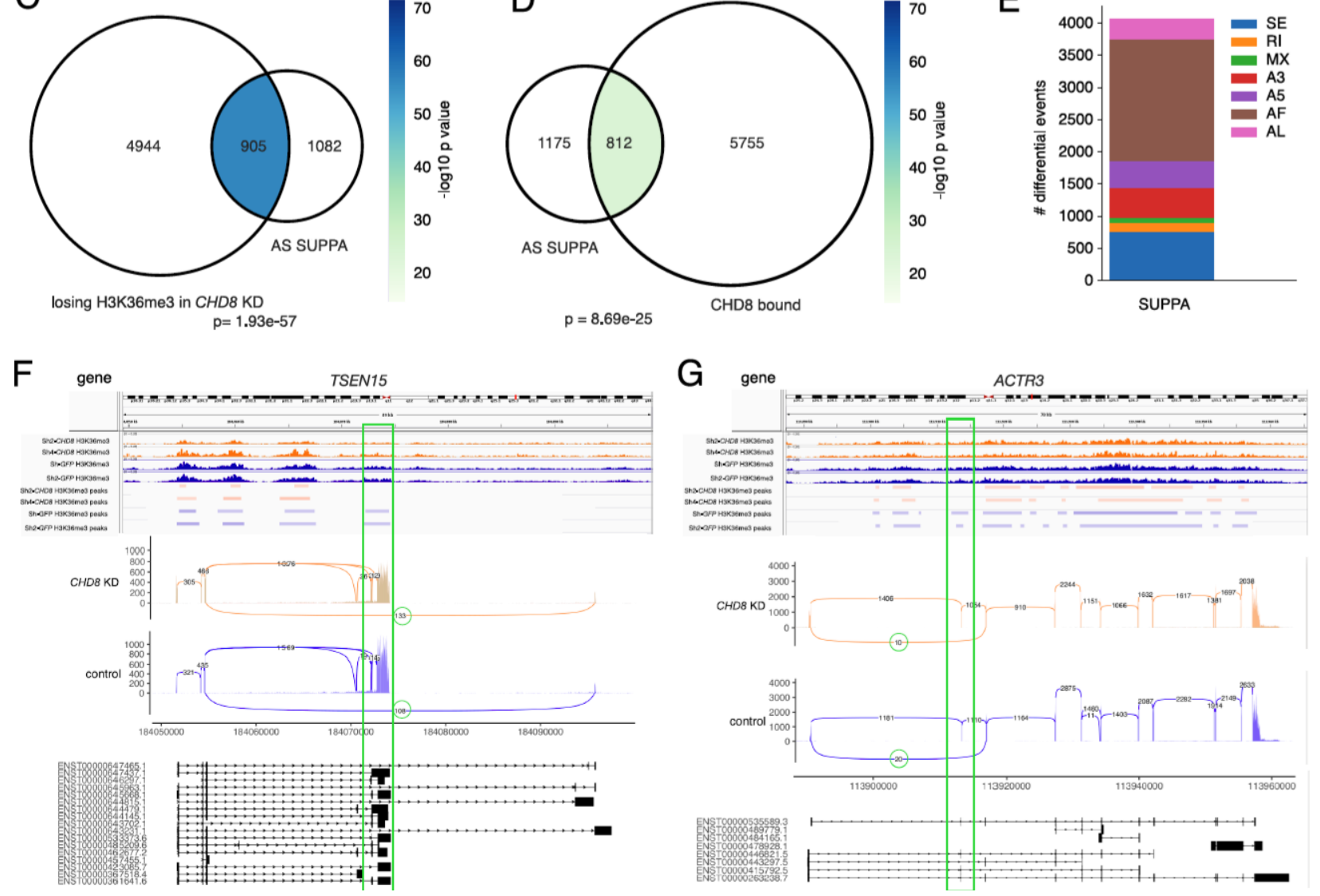
Figure 4 CHD8-suppression elicited reduction in H3K36me3 correlates with significant alterations in RNA alternative splicing.

A. B. The box plots show the RNA expression level average of protein coding genes (A) and for genes that lose H3K36me3 peaks following CHD 8 knock-down (B). Control condition is shown in dark grey, $C H D 8$ knock-down in white; genes bound (left) and unbound by CHD8 (right) are also indicated. TPM: Transcripts Per Kilobase Million. Extreme outliers not shown. ns = not significant, $\mathrm{t}$ test statistic.

C. D. Venn diagrams represent the overlap between genes losing H3K36me3 peaks following CHD8 knock-down (losing H3K36me3 in CHD8 knock-down) and genes presenting altered alternative splicing events as detected by SUPPA (AS SUPPA) (C), and the overlap between genes bound by CHD8 (CHD8-bound) and genes presenting altered alternative splicing events as detected by SUPPA (AS SUPPA). Number of genes for each condition is indicated. The enrichment significance for each intersection is computed by Fisher's exact test and represented by colors. Color coded legend: $-\log 10$ ( $\mathrm{p}$ value).

E. Stacked bar plot represents the 1987 differential alternative splicing events detected by SUPPA, distributed by event type. SE, skipped event; RI, retained intron; MX, mixed event; A3, alternative 3'; A5, alternative 5'; AF, alternative first exon; AL, alternative last exon.

F. G. Data from two representative genes are shown along with their genomic sites, TSEN15 (G) and ACTR3 (F), including H3K36me3 ChIP-seq tracks for CHD8 knock-down (Sh2-CHD8 and Sh4-CHD8 in orange) and control (Sh-GFP and Sh-GFP2 in blue), with the corresponding peaks identified by MACS2 (top). At the bottom, sashimi plots display the alternative last (AL) event for TSEN15 (G) and skipped exon (SE) for ACTR3 (F), corresponding to the H3K36me3 peak 
bioRxiv preprint doi: https://doi.org/10.1101/2020.03.14.992032. The copyright holder for this preprint (which was not peer-reviewed) is the author/funder. All rights reserved. No reuse allowed without permission.

lost after $C H D 8$ suppression (highlighted by green rectangles), in orange for CHD 8 knock-down and in blue for control. 